\title{
Assessments of $k-k L$ Turbulence Model Based on Menter's Modification to Rotta's Two-Equation Model
}

\author{
Khaled S. Abdol-Hamid \\ NASA Langley Research Center, Hampton, VA 23693, USA \\ Correspondence should be addressed to Khaled S. Abdol-Hamid; k.s.abdol-hamid@nasa.gov
}

Received 25 July 2014; Revised 6 December 2014; Accepted 7 December 2014

Academic Editor: James J. McGuirk

Copyright (C) 2015 United States Government as represented by the Administrator of the National Aeronautics and Space Administration. No copyright is claimed in the United States under Title 17, U.S. Code. All Other Rights Reserved.

\begin{abstract}
The main objective of this paper is to construct a turbulence model with a more reliable second equation simulating length scale. In the present paper, we assess the length scale equation based on Menter's modification to Rotta's two-equation model. Rotta shows that a reliable second equation can be formed in an exact transport equation from the turbulent length scale and kinetic energy. Rotta's equation is well suited for a term-by-term modeling and shows some interesting features compared to other approaches. The most important difference is that the formulation leads to a natural inclusion of higher order velocity derivatives into the source terms of the scale equation, which has the potential to enhance the capability of Reynolds-averaged Navier-Stokes to simulate unsteady flows. The model is implemented in the CFD solver with complete formulation, usage methodology, and validation examples to demonstrate its capabilities. The detailed studies include grid convergence. Near-wall and shear flows cases are documented and compared with experimental and large eddy simulation data. The results from this formulation are as good or better than the well-known shear stress turbulence model and much better than $k-\varepsilon$ results. Overall, the study provides useful insights into the model capability in predicting attached and separated flows.
\end{abstract}

\section{Introduction}

While two-equation models have been used routinely to simulate turbulence flows for the last 50 years, they are based on a kinetic energy equation and either dissipation or timescale equation to evaluate length scale. These two scales are obtained from the solution of two presumably independent transport equations, like the $k-\varepsilon$ or $k-\omega$ model or any other formulations that use a $k$ equation. The mechanism of the second equation for determining a turbulent length scale is not fully understood, and a number of formulations use a special boundary condition for simulating wall boundary conditions. Even the more complex model closures like Reynolds stress models (RSM) or explicit algebraic Reynolds stress models (EARSM) are still using length scale equations based on an underlying two-equation model. Almost all twoequation models use the turbulent kinetic energy, $k$, and its transport equation as one of the primary variables. The exact transport equation for $k$ can be modeled with a few relatively straightforward assumptions.

Historically, the modeling of the second equation is therefore purely heuristic using dimensional arguments [1]. These models use strain-rate or vorticity derived from the mean flow resulting in only one scale from the equilibrium of source terms for both equations. In fact, the second equation is considered, in most cases, the weakest link when using turbulence models, including much more complex approaches such as full Reynolds stress and hybrid Reynoldsaveraged Navier-Stokes (RANS)/large eddy simulation (LES) formulations. It is very difficult to justify using any of the complex turbulence models without fixing or using a better form for the second equation. The main objective of the present paper is to assess a better form for the second equation. One of the limited exceptions is the modeling concept proposed by Rotta [2], which can be formed in an exact transport equation for the turbulent length scale, $L$. Rotta's 
equation is well suited for a term-by-term model and shows some interesting features compared to other approaches. The most important difference is that the formulation leads to a natural inclusion of higher-order velocity derivatives into the source terms of the scale equation. This enhances the resolution of the turbulent spectrum in unstable flow conditions.

Menter et al. [3-5] presented a complete form of a $k-\sqrt{k} L$ two-equation turbulence model based on the Rotta model [2]. Menter et al. [3-5] replaced problematic third velocity derivatives originally formulated by Rotta [2] in the second equation with second velocity derivatives. They utilize this two-equation turbulence model to formulate a scaleadaptive simulation (SAS) term that can be added to other two-equation turbulence models such as the shear stress turbulence model (SST). The SAS concept is based on the introduction of the von Karman length-scale into the turbulence scale equation. The information provided by the von Karman length scale allows SAS models to dynamically adjust into resolved structures in an unsteady Reynolds-averaged Navier-Stokes (URANS) simulation, which results in a LESlike behavior in unsteady regions of the flowfield. At the same time, the model provides standard RANS capabilities in stable flow regions.

In the present paper, a complete turbulence model form based on Rotta's $k$ - $k L$ two-equation model is implemented in the computational fluid dynamics (CFD) PAB3D solver. The complete model does not use a blending function to merge two turbulence scale equations, as is done with SST or damping functions used by $k-\varepsilon$, and naturally contains the SAS characteristics through the van Karman length-scale. The formulations, usage methodology, and validation examples demonstrate the capabilities of the present $k-k L$ turbulence model. The model provides proper RANS performance in stable flow regions and allows the break-up of large turbulent structures for unstable flow regimes (e.g., a cylinder in cross flow and flow in cavities). Solutions are compared with other forms of RANS results, LES data, and experimental data for different flows.

Results for all near-wall and shear flows have been calculated using the structured multiblock grids, resolving the viscous sublayer with $y+<1$. All simulations have been carried out on highly refined grids, avoiding any grid refinement uncertainties. The results are compared with other turbulence models and available experimental or theoretical data. Most of the cases are taken from the cases compiled in the turbulence modeling resource webpage [6]. The purpose of this site is to provide a central location where RANS turbulence models are documented. Table 1 presents a list of validation cases that will be discussed in detail in the paper.

\section{Turbulence Models}

The present $k$ - $k L$ two-equation turbulence model, listed here, is based on the approach made by Menter et al. [3-5] to develop the $k-\sqrt{k} L$ model. The compressibility correction function has been added for high-speed flow. The model is based on Rotta's $k-k L(\Phi=k L)$ with modifications made by
Menter in which the velocity third derivative is replaced by a second derivative. The model is casted in $k-\Phi$ form for direct comparisons with $k-\sqrt{k} L$ as follows:

$$
\begin{aligned}
\frac{\partial \rho k}{\partial t}+ & \frac{\partial \rho U_{j} k}{\partial x_{j}} \\
= & {\left[P_{k}+\frac{\partial}{\partial x_{j}}\left(\left[\mu_{l}+\frac{\mu_{t}}{\sigma_{k}}\right] \frac{\partial k}{\partial x_{j}}\right)+\mathrm{VSM}_{k}\right] } \\
& -\left[C_{D} \rho \frac{k^{p 1}}{\Phi}\right], \\
\frac{\partial \rho \Phi}{\partial t}+ & \frac{\partial \rho U_{j} \Phi}{\partial x_{j}} \\
= & {\left[C_{\phi 1} \frac{\Phi}{k} P_{k}+\frac{\partial}{\partial x_{j}}\left(\left[\mu_{l}+\frac{\mu_{t}}{\sigma_{\Phi}}\right] \frac{\partial \Phi}{\partial x_{j}}\right)+\mathrm{VSM}_{\Phi}\right] } \\
& -C_{\phi 2} \rho k^{p 1-1},
\end{aligned}
$$

where

$$
\begin{gathered}
C_{\phi 1}=\left(\zeta_{1}-\zeta_{2}\left(\frac{L}{L_{v k}}\right)^{2}\right), \quad C_{\phi 2}=\left(\zeta_{3}+p_{1} C_{\mu}^{3 / 4} f_{c}\right), \\
C \mu=0.09, \quad C_{D}=C_{\mu}^{3 / 4}\left(1 .+f_{c}\right) .
\end{gathered}
$$

The production is modeled as $C$

$$
P_{k}=-u_{i} u_{j} \frac{\partial u_{i}}{\partial x_{j}}, \quad \mu_{t}=C_{\mu}^{1 / 4} \frac{\rho \Phi}{k^{p 1-2}} .
$$

The von Karman length scale, $L_{v k}$, is defined as

$$
L_{v k}=\kappa\left|\frac{U^{\prime}}{U^{\prime \prime}}\right|
$$

where

$$
\begin{gathered}
U^{\prime}=S=\sqrt{2 S_{i j} S_{i j}}, \\
S_{i j}=\frac{1}{2}\left(\frac{\partial U_{i}}{\partial x_{j}}+\frac{\partial U_{j}}{\partial x_{i}}\right), \\
U^{\prime \prime}=\sqrt{\left(\frac{\partial^{2} U_{i}}{\partial x_{k}^{2}} \frac{\partial^{2} U_{i}}{\partial x_{j}^{2}}\right) .}
\end{gathered}
$$

To avoid overly large or small values of the length scale ratio, $L / L_{v k}$, we set $L / C_{11}<L_{v k}<C_{12} k d$, where $d$ is the distance from the nearest wall, $C_{l 1}=10$, and $C_{l 2}=1.3$. The upper limit in the von Karman length scale is based on the attached flow assumption that it is directly proportionate to the distance from the wall. This assumption is not true for separated flow. We are proposing to reduce the upper limit in the separation region using the production $(P)$ to dissipation (c) ratio:

$$
\frac{L}{C_{l 1}}<L_{v k}<C_{l 2} \kappa d\left[\frac{P}{C_{\mu}^{3 / 4} \rho} \frac{\Phi}{k^{p 1}}\right] .
$$


TABLE 1: Unit test cases representing steady/unsteady, attached/separated, and wall/shear flows.

\begin{tabular}{lrc}
\hline Case & Details and references & Flow characteristics \\
\hline A. & 2D zero pressure gradient flat plate [6] compared data from [6] & Attached/steady \\
B. & 2D backward-facing step [6] compared with data from [7] & Separated/unsteady \\
C. & Flow over a hump model [8] compared with data from [8-10] & Separated/unsteady \\
D. & Axisymmetric subsonic jet [6] compared with experimental data from [11] & Steady/shear \\
E. & 2D curved backward-facing step [6] will be compared with LES data from [12] & Separated/unsteady \\
F. & 2D periodic hill [6] compared with LES data from [13] & Separated/unsteady \\
G. & 2D NACA 4412 Airfoil trailing edge separation [6] compared with data from [14] & Separated/steady \\
H. & Stationary circular cylinder in cross flow with data from [15] & Wake/unsteady \\
\hline
\end{tabular}

The length scale, $L$, is calculated from the following equation:

$$
L=\frac{\Phi}{k^{p 1-1.5}}
$$

To integrate the model through the viscous sublayer, additional near wall damping terms are required. The following terms are added to the right hand sides of the $k$ and the $\Phi$ equation, respectively:

$$
\begin{gathered}
\operatorname{VSM}_{k}=-2 \mu_{l} \frac{k}{d^{2}} \\
\operatorname{VSM}_{\Phi}=-6 \mu_{l} \frac{\Phi}{d^{2}} f_{\Phi}
\end{gathered}
$$

where

$$
\begin{gathered}
f_{\Phi}=\frac{1+C_{d 1} \xi}{1+\xi^{4}}, \\
\xi=\frac{\sqrt{0.3 k} d}{20 \nu_{l}} .
\end{gathered}
$$

To close the turbulence model, [3] used the following logarithmic requirements:

$$
\begin{gathered}
\frac{d U}{d y}=\frac{u_{\tau}}{\kappa y}, \quad k=\frac{u_{\tau}^{2}}{C_{\mu}^{1 / 2}}, \\
L=\kappa y, \quad v_{t}=u_{\tau} \kappa y .
\end{gathered}
$$

Then,

$$
\zeta_{2}=\zeta_{1}-\zeta_{3} \frac{1}{C_{\mu}^{3 / 4}}+\frac{\kappa^{2}}{\sigma_{\phi} C_{\mu}^{1 / 2}}
$$

The final set of constants and coefficients for the $k-\sqrt{k} L$ turbulence model is listed from [3]:

$$
\begin{aligned}
& \text { for } \Phi=\sqrt{k} L, \\
& p 1=2, \\
& \zeta_{1}=0.8,
\end{aligned}
$$

$$
\begin{aligned}
\zeta_{2} & =1.47, \\
\zeta_{3} & =0.0288, \\
\sigma_{k} & =\frac{2}{3}, \\
\sigma_{\Phi} & =\frac{2}{3} .
\end{aligned}
$$

To come up with the present set of constants and coefficients for the present $k-k L$ :

(a) use the logarithmic requirements;

(b) limit the range for $1.1<\zeta_{1}<1.3$ and $0.95<\zeta_{2}<1.0$ as suggested by Rotta [2];

(c) calibrate both coefficients for the best results for cases $\mathrm{A}$ and $\mathrm{D}$ from the present paper.

Below is the final list of constants and coefficients used in the present paper for the $k-k L$ turbulence model:

$$
\begin{aligned}
& \text { for } \Phi=k L, \\
& p 1=2.5, \\
& \zeta_{1}=1.2, \\
& \zeta_{2}=0.97, \\
& \zeta_{3}=0.13, \\
& \sigma_{k}=1.0, \\
& \sigma_{\Phi}=1.0 .
\end{aligned}
$$

\section{Results and Discussions}

3.1. 2D Zero Pressure Gradient Flat Plate Case. Figure 1 shows the sketch of the flat plate test case with boundary conditions used in this analysis. This is a subsonic $M=0.2$ case at $\operatorname{Re}=5$ million based on unit length. The plot in Figure 2 shows the convergence of the wall skin friction coefficient at $x=0.97$ using five levels of grid size with the $k-k L$ turbulence model. Each coarser grid is exactly every-otherpoint of the next finer grid, ranging from the super fine grid of $544 \times 384$ cells to the very coarse grid of $34 \times 24$ cells. In 


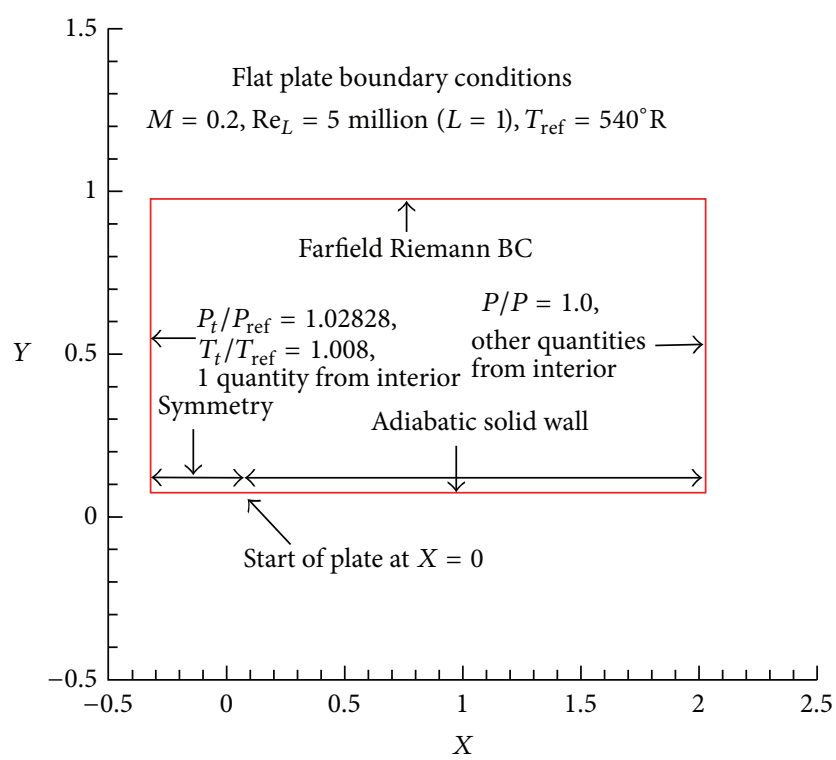

FIGURE 1: Sketch of $M=0.2, \operatorname{Re}=5$ million flat plate test case [6].

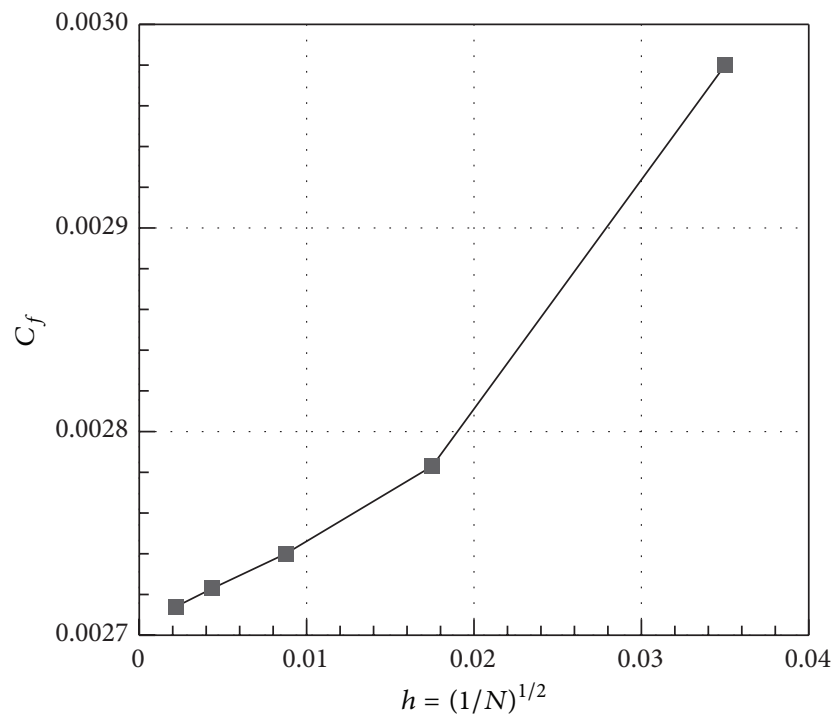

FIGURE 2: Skin friction coefficient variation with grid refinement using the $k-k L$ turbulence model (PAB3D) at $x=0.97$.

the plot, the $x$-axis is plotting $(1 / N)^{1 / 2}$, which is proportional to grid spacing $(h)$. At the left of the plot, $h=0$ represents an infinitely fine grid. The difference between the coarsest and finest grid is less than 0.0003 in skin friction, but the very coarse grid is out of order from the other levels. The surface skin friction coefficient, using the $k-k L$ turbulence model on the finest $544 \times 384$ grid cells over the entire plate, varies with respect to momentum thickness Reynolds number, as shown in Figure 3. The $k-k L$ results are in good comparisons with the SST solution computed using the CFL3D code [16].

Figure 4 shows the grid effects in predicting $u+$ using coarse, medium, fine, and super fine grids. All the grid levels predicted similar $u+$ variations with $y+$ except for

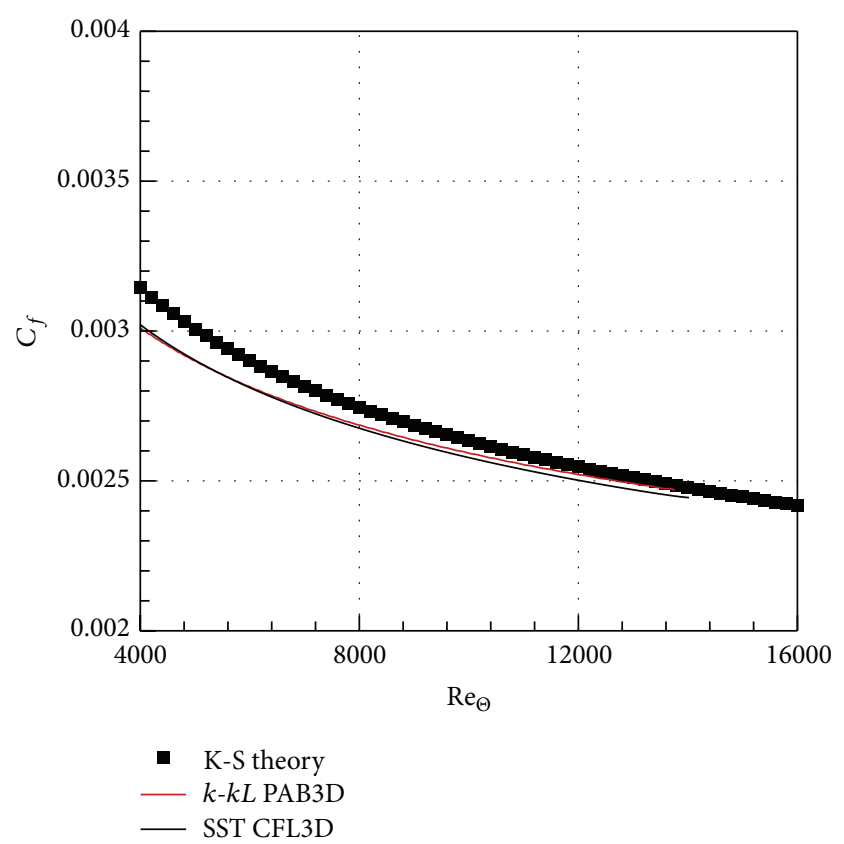

FIGURE 3: Flat plate skin friction coefficient variations with $\mathrm{Re}_{\Theta}$ showing comparisons between $k-k L$ (PAB3D), SST (CFL3D), and K-S theory.

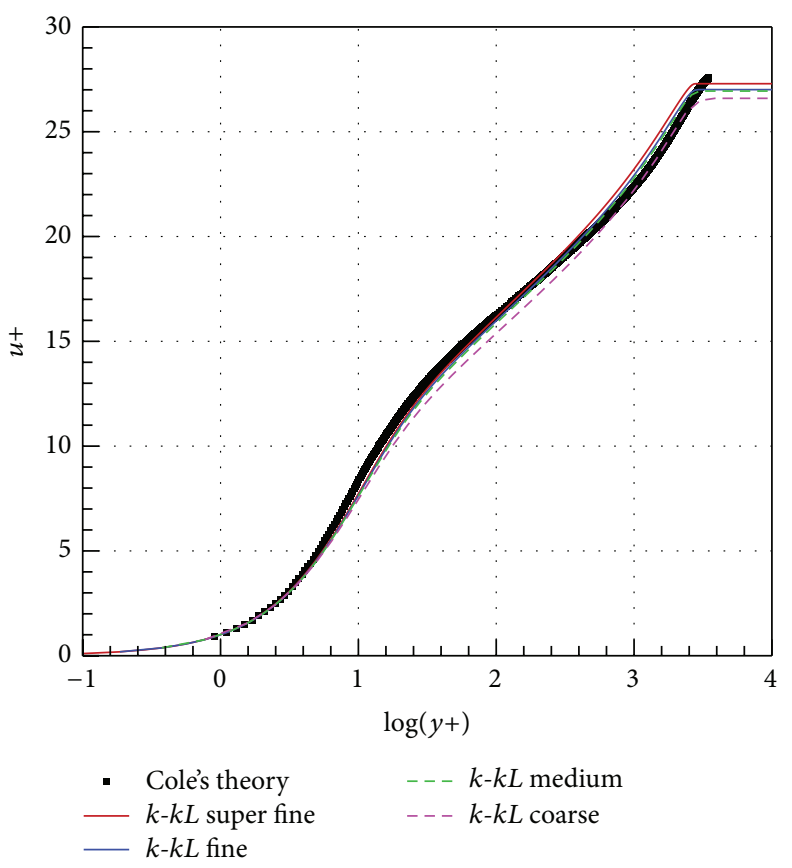

FIGURE 4: Flat plate $u+-y+$ variations with comparisons using $k-k L$ (PAB3D) with different grid levels and Coles' Theory at $x=0.97$.

the coarse grid that slightly underpredicted these variations. Figure 5 shows the variation of $u+$ velocity with respect to $y+$ predicted using $k-k L$, SST, and $k-\varepsilon$ turbulence models using the finest grid results. The $k-k L$ results are in very good comparisons with SST and Coles' data taken from [6]. The $k-\varepsilon$ results, as expected, largely underpredicted the $u+$ levels 


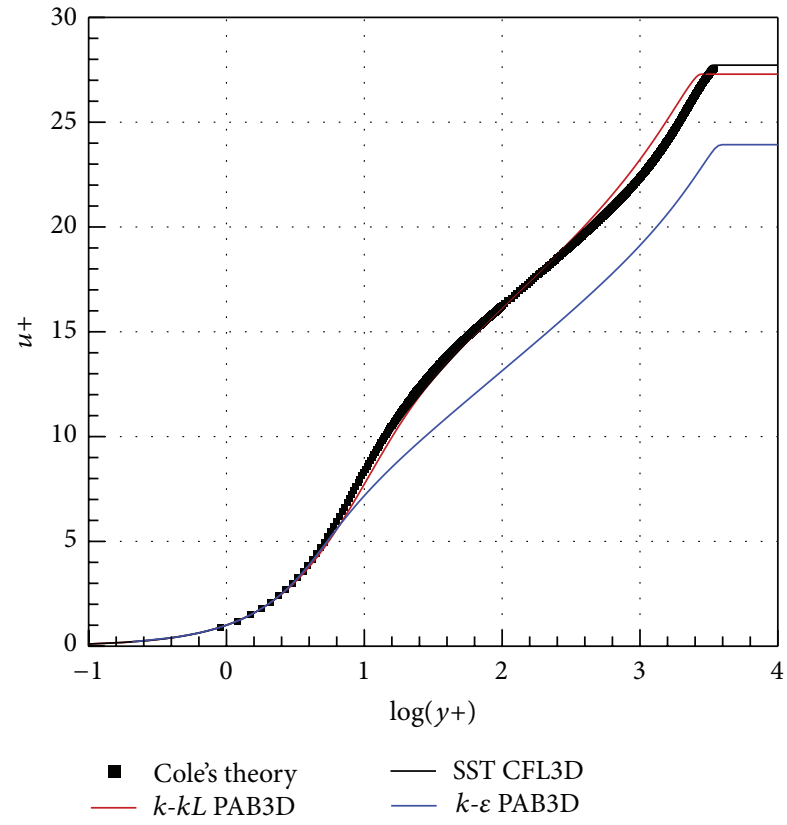

FIgURE 5: Flat plate $u+-y+$ variations with comparisons between $k-k L$ (PAB3D), $k-\varepsilon$ (PAB3D), SST (CFL3D), and Cole's theory at $x=$ 0.97 .

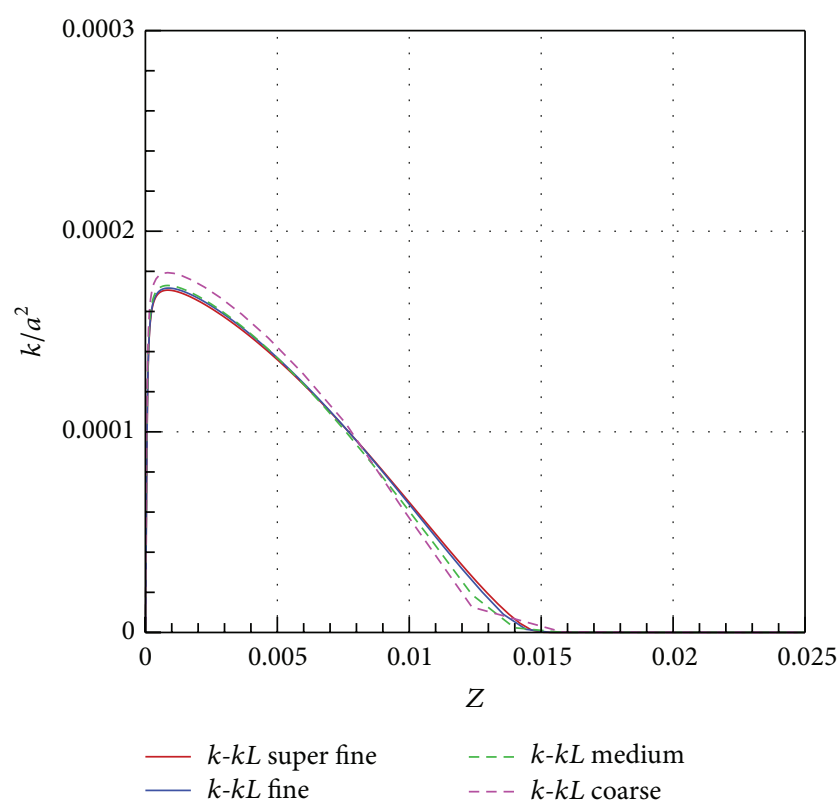

FIGURE 6: Flat plate $k / a^{2}$ variations with comparisons between $k-k L$ (PAB3D) with different grid levels at $x=0.97$.

through the entire $y+$ range, which are caused by predicting much higher surface skin friction than $k-k L$ or SST models.

Turbulence kinetic energy and viscosity are other quantities used to validate the quality of turbulence model predictions. Figure 6 shows the grid convergence in the prediction of the kinetic energy values at $x=0.97$. Only the coarse grid shows larger values of turbulence kinetic energy up to $Z=$ 0.01 . The medium, fine, and superfine grid levels predicted

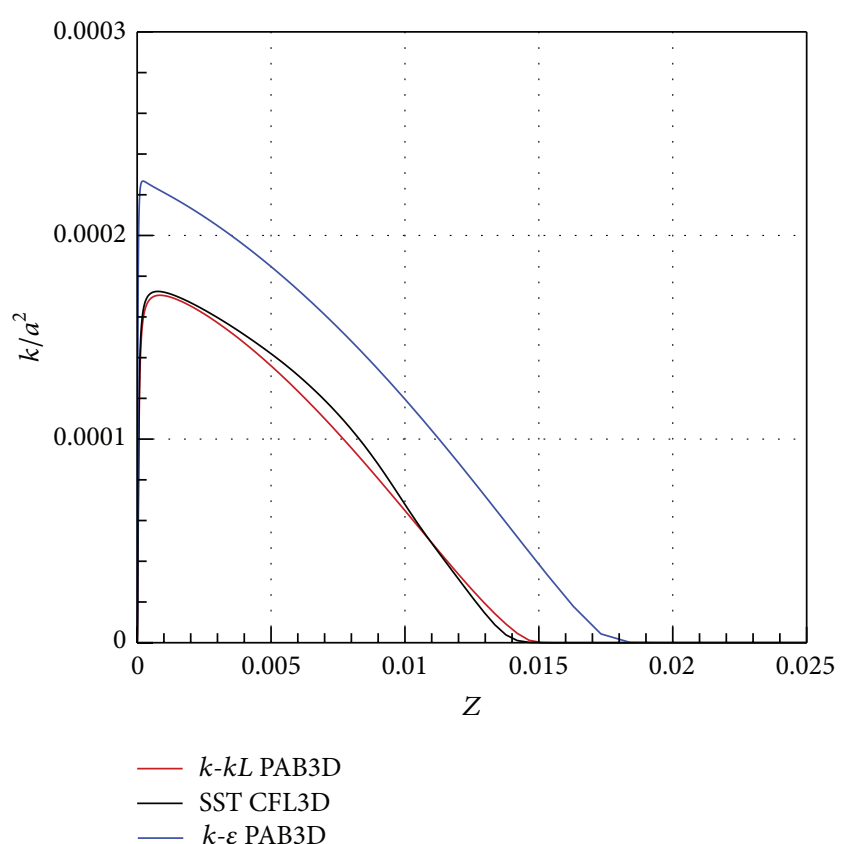

FIGURE 7: Flat plate $k / a^{2}$ variations with comparisons between $k-k L$ (PAB3D), $k-\varepsilon$ (PAB3D), and SST (CFL3D) at $x=0.97$.

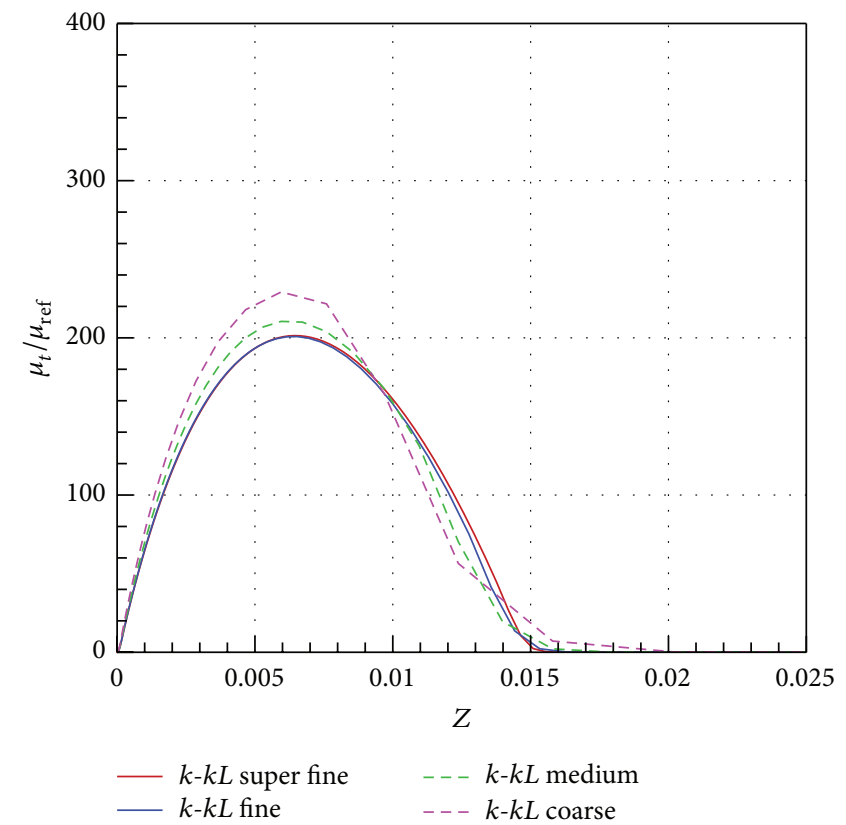

FIGURE 8: Flat plate turbulence viscosity variations with comparisons between $k-k L$ (PAB3D), $k-\varepsilon$ (PAB3D), and SST (CFL3D) at $x=0.97$.

very similar turbulence kinetic energy distribution. Figure 7 shows comparisons between the predicted turbulence kinetic energy using $k-k L$, SST, and $k-\varepsilon$ turbulence models. The $k-k L$ and SST results are in very good agreement, however, the $k-\varepsilon$ results show much higher turbulence kinetic energy than either of the other two models. Figure 8 shows the grid convergence in the prediction of turbulence viscosity. Similar 


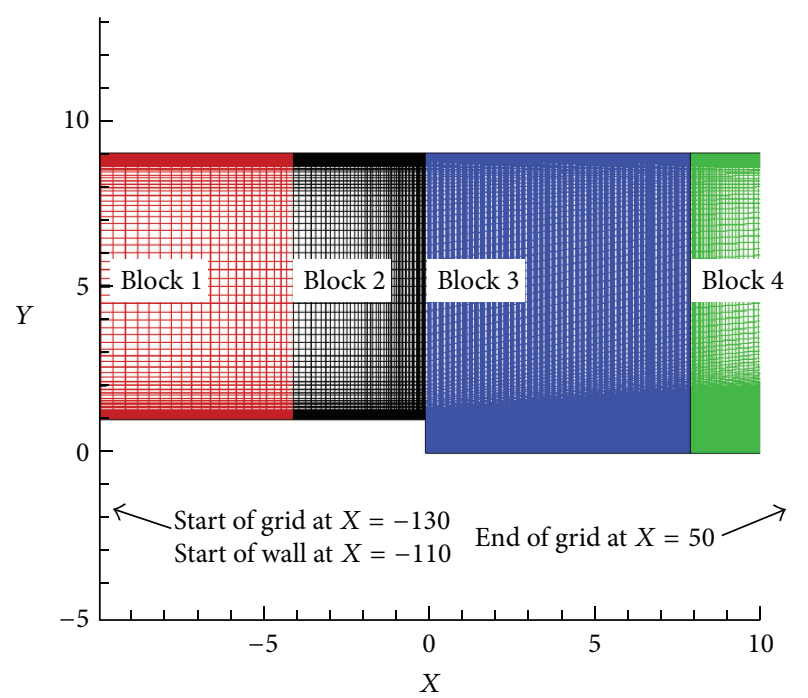

FIGURE 9: Grid distribution for the backward-facing step grid [6].

to the observation made for the kinetic energy, the coarse grid predicted much higher turbulence viscosity than other grids. This happens because the coarse grid has less than 50 cells in the normal direction and less than 25 cells to capture the entire boundary layer.

3.2. 2D Backward-Facing Step Case. The 2D backward-facing step case is a backward-step flow case with a sharp corner. A series of 2D grids, nondimensionalized by the step height $H$, are used in this analysis taken from [6]. As structured grids, these are comprised of four zones connected in a one-to-one fashion. Each coarser grid is exactly every-other-point of the next finer grid, ranging from the finest cells, $256 \times 256,96 \times$ $256,384 \times 448$, and $128 \times 448$, to the coarsest cells, $64 \times 64,24$ $\times 64,96 \times 112$, and $32 \times 112$. Figure 9 shows a portion of the coarse grid distribution. In this case, a turbulent boundary layer encounters a sudden back step, causing flow separation. The flow then reattaches and recovers downstream of the step. The Reynolds number based on boundary layer momentum thickness prior to the step is 5000. This corresponds to a Reynolds number of approximately 36,000 based on step height $H$. The boundary layer thickness prior to the step is approximately $1.5 \mathrm{H}$. The flow conditions for this case are

$$
\begin{gathered}
M=0.128, \quad \operatorname{Re}=36,000 \text { (based on step height } H), \\
T=537^{\circ} \mathrm{R} .
\end{gathered}
$$

One of the key measures of success for this flowfield of turbulence models is the prediction of separation and reattachment points downstream of the step. In experiment [7], this was determined by laser oil-flow interferometer measurements of skin friction and interpolation of the zero skin-friction location. The experimental reattachment point is around $6.26 \pm 0.1$. The $U_{\text {ref }}$ is the reference velocity at the center-channel near $x / H=-4$ (where $H=1$ grid unit). The coarse grid solution using the $k-k L$ model is steady, where

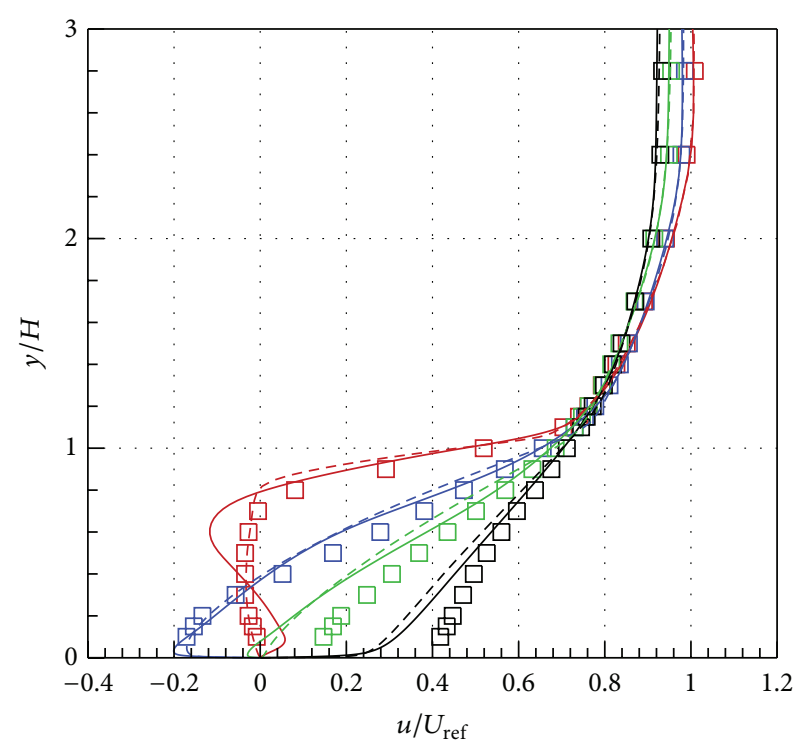

$$
\begin{array}{ll}
\square \text { Exp, } x / H=1 & \quad \text { Exp, } x / H=10 \\
\square \text { Exp, } x / H=4 & -k-k L \text { modified, } x / H=1 \\
\square \text { Exp, } x / H=6 & ---k-k L \text { original, } x / H=1
\end{array}
$$

FIGURE 10: Velocity distribution comparisons between experimental data and original (dashed line) and modified $k-k L$ (solid line) predictions for backward-facing step case.

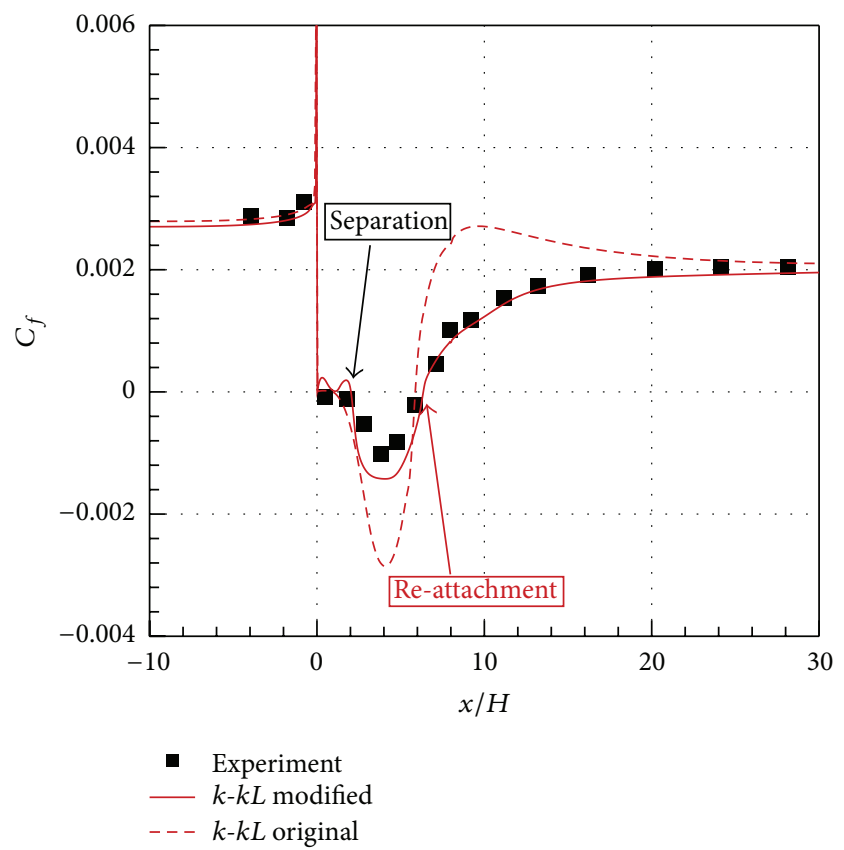

FIGURE 11: Skin friction comparisons between experimental data and original and modified $k-k L$ predictions for backward-facing step case.

the other two grid levels are showing unsteady behavior, as shown later in Figure 12. The time-average of the unsteady data for all cases is used to compare with experimental data. Next, we evaluate the correction made for the upper limit of the von Karmen length scale (Equation (6)), referred to as the modified $k-k L$ model. Figure 10 shows the comparison 


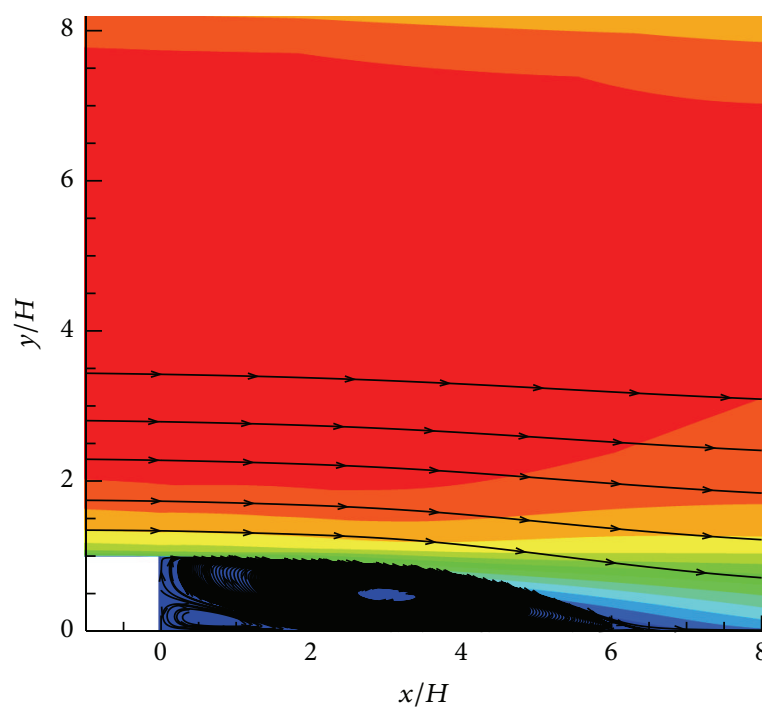

(a) Coarse grid (steady-state)

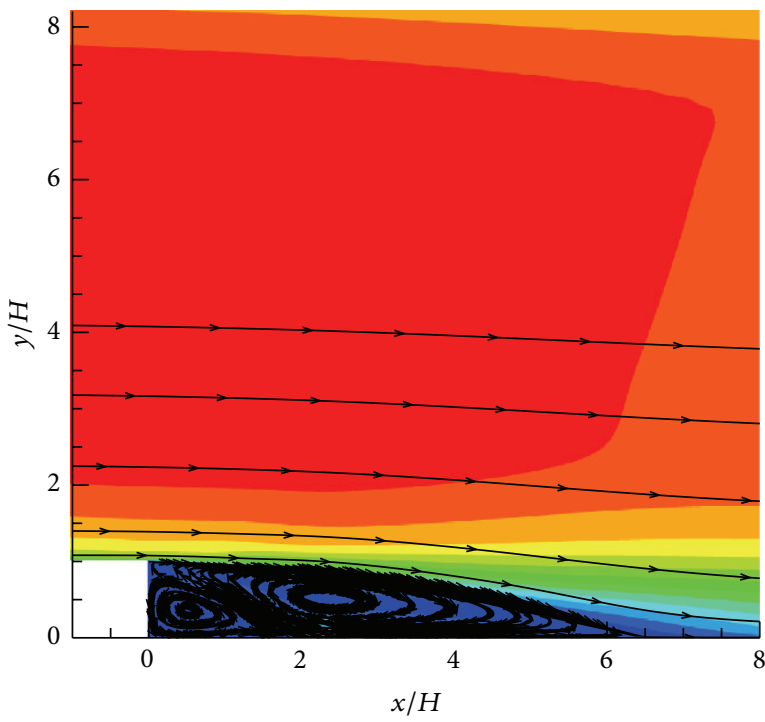

(c) Fine grid (time-averaged)

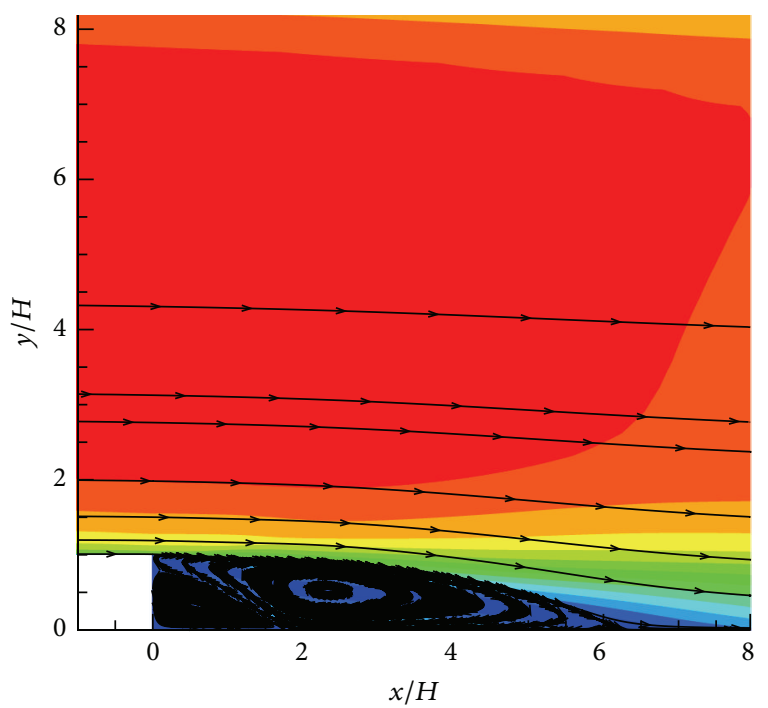

(b) Medium grid (time-averaged)

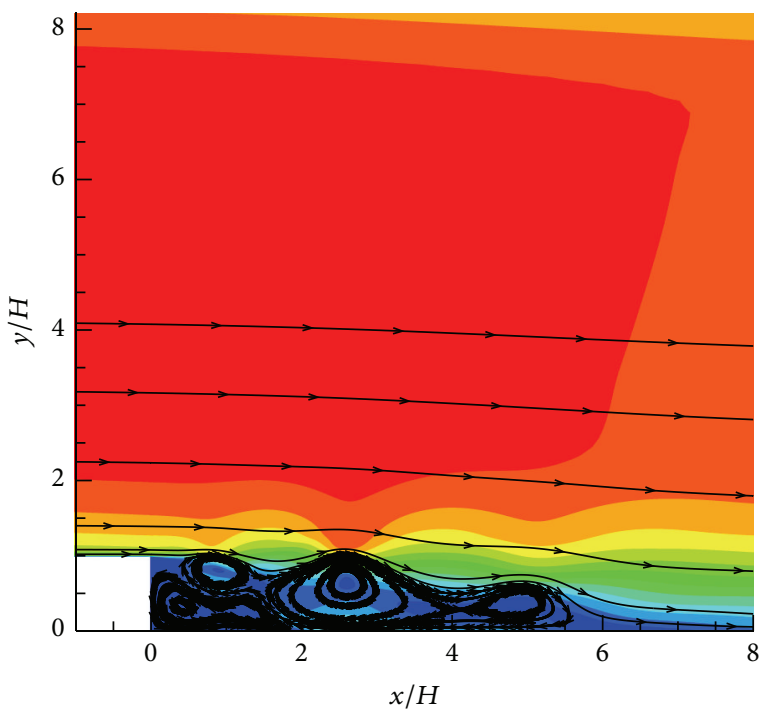

(d) Fine grid (unsteady)

FIGURE 12: Streamlines predictions using the $k-k L$ turbulence model at different grid levels for backward-facing step case.

of velocity distribution predictions at different $x / H$ locations using the original and modified $k-k L$ models. There is no significant difference between the prediction using both models with the exception of the velocity distribution at $x / H=1$. The skin friction results in Figure 11 show more significant differences between the predictions from both models. In the region of attached flow up to $x / H=2$, there is no difference between both models as the production to dissipation ratio is around 1.0 in the attached flow region. In the separated flow region, the ratio is much smaller than 1 , which causes a significant difference in skin friction predictions. The original model clearly underpredicts the skin friction in the separated flow region and overpredicts the skin friction in the region downstream of the reattachment point. The modified $k-k L$ model results are in very good agreement with the experimental data.
Figures 12(a), 12(b), and 12(c) show the time-averaged streamline results from the coarse, medium, and fine grid solutions. All solutions show two bubbles: one close to the corner of the step where the flow starts to separate and the other which contains the large separation bubble. The coarse grid produces a smaller first bubble and larger second bubble with the reattachment location around $x / H=6.78$. The medium and fine grids produce very similar bubble sizes, and the reattachment location is predicted at $x / H=6.3$, as shown in Figure 13.

Figure 13 shows the comparisons between skin friction predictions using coarse, medium, and fine grids. The medium and fine grid simulations are in good agreement with experimental data predicting the separation and reattachment locations. Figure 14 shows the skin friction comparisons between experimental data $k-k L$, SST, and $k-\varepsilon$ 


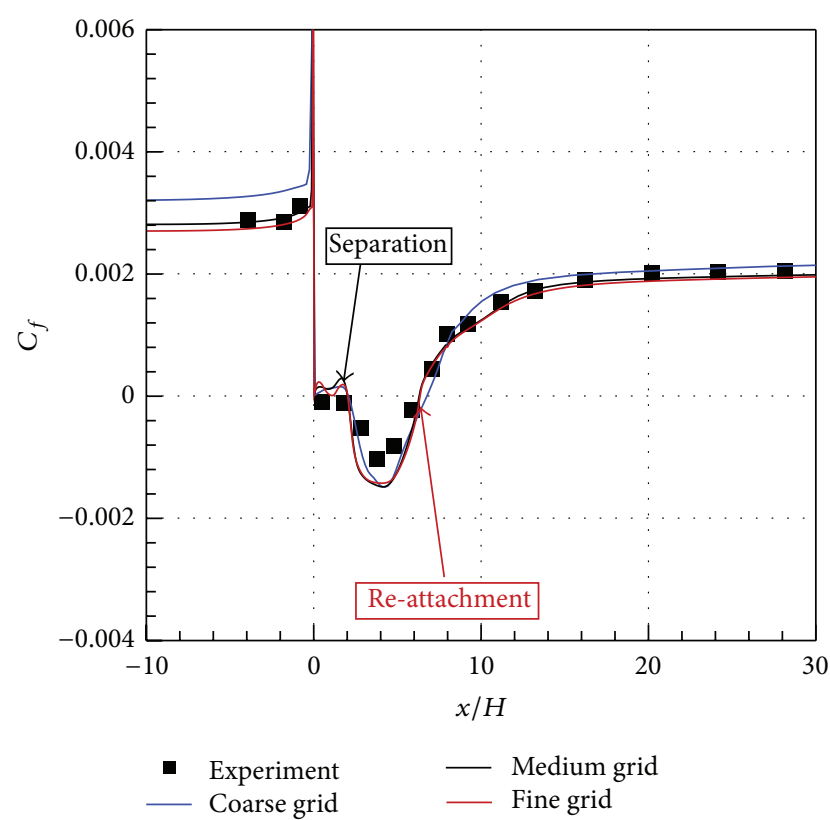

FIGURE 13: Skin friction comparisons between experimental data and coarse, medium, and fine grid simulations for backward-facing step case.

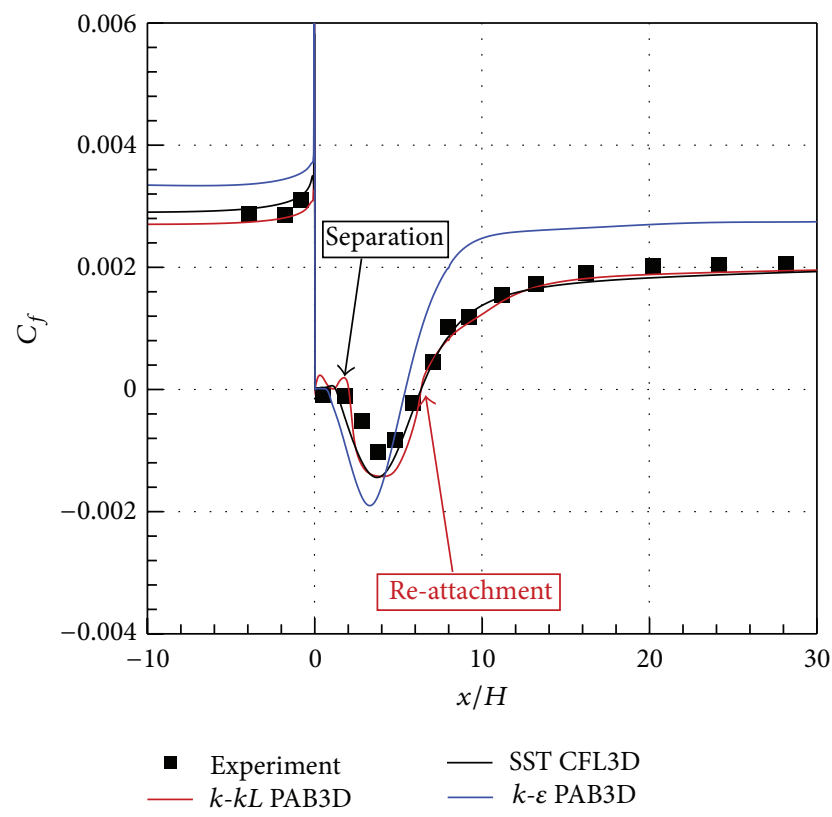

FIGURE 14: Skin friction comparisons between experimental data and $k-k L$, SST, and $k-\varepsilon$ simulations for backward-facing step case.

simulations. The $k-\varepsilon$ simulation predicts early separation and a reattachment location around $x / H=5.4$, compared to experimental data of 6.26 . The $k-k L$ is slightly better than SST in predicting skin friction and reattachment point prediction. Figure 15 shows the velocity distribution comparisons between experimental data, and $k-k L$ and SST simulations. The SST matches the experimental data better at $x / H=1$ compared to the $k-k L$ simulation. However, the $k-k L$ is in

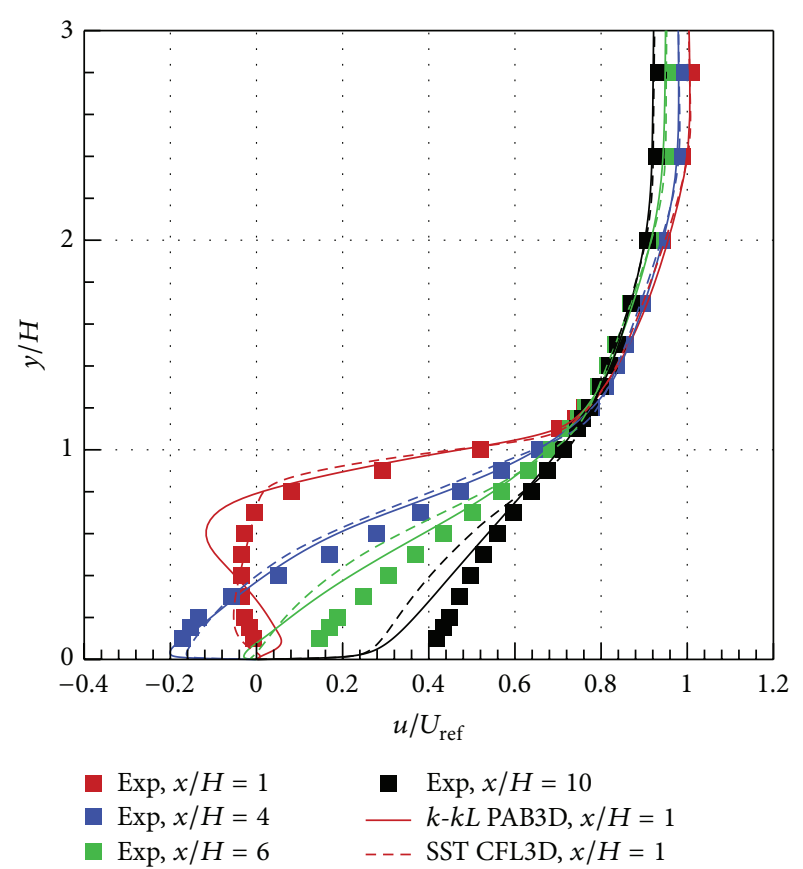

FIGURE 15: Velocity distribution comparisons between experimental data and $k-k L$ and SST simulations for backward-facing step case.

closer agreement with the experimental data for $x / H=6$ and 10 locations.

3.3. 2D Hump Case. Case 3, from the CFD 2004 validation workshop for Synthetic Jets and Turbulent Separation [8] is a wall-mounted Glauert-Goldschmied type. The model itself is 23 inches wide between the endplates at both sides (each endplate is approximately 9.25 inches high, 34 inches long, and 0.5 inches thick with an elliptical-shaped leading edge). The model is 2.116 inches high at its maximum thickness point. In the present paper, we simulate the (baseline) case with flow conditions of $M=0.1$ and a Reynolds number of approximately 1 million per chord. Only surface pressure and skin friction are compared with results from turbulence model simulations. Three grid levels are utilized to assist grid convergence; the finest grid consists of two zones of $416 \times 216$ and $416 \times 216$ cells, and the coarsest has $104 \times 54$ and $104 \times$ 54 cells. This is based on structured 2D grid no. 5 from [9] as it is built with the top wall shape adjusted to approximately account for side plate blockage. Figure 16 shows a portion of the coarse grid distribution. In this case, the boundary layer is subjected to a favorable pressure gradient over the front convex portion of the body and separates over a relatively short concave section in the aft part of the body. The flow then reattaches and recovers downstream of the hump.

Surface pressure results from the modified and original $k-k L$ turbulence models are compared with experimental data from surface mounted pressure taps [10], as shown in Figure 17. Both models compared well with experimental data with a slight improvement using the original model at $x=0.8$. However, the skin friction prediction from the modified model is in pretty good agreement with experimental 


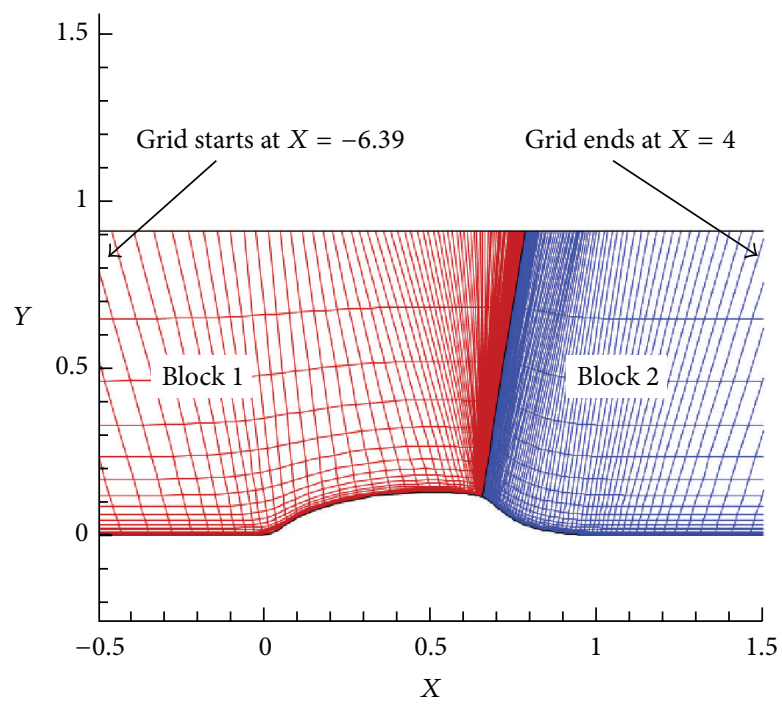

FIGURE 16: Grid distribution for the subsonic hump model case.

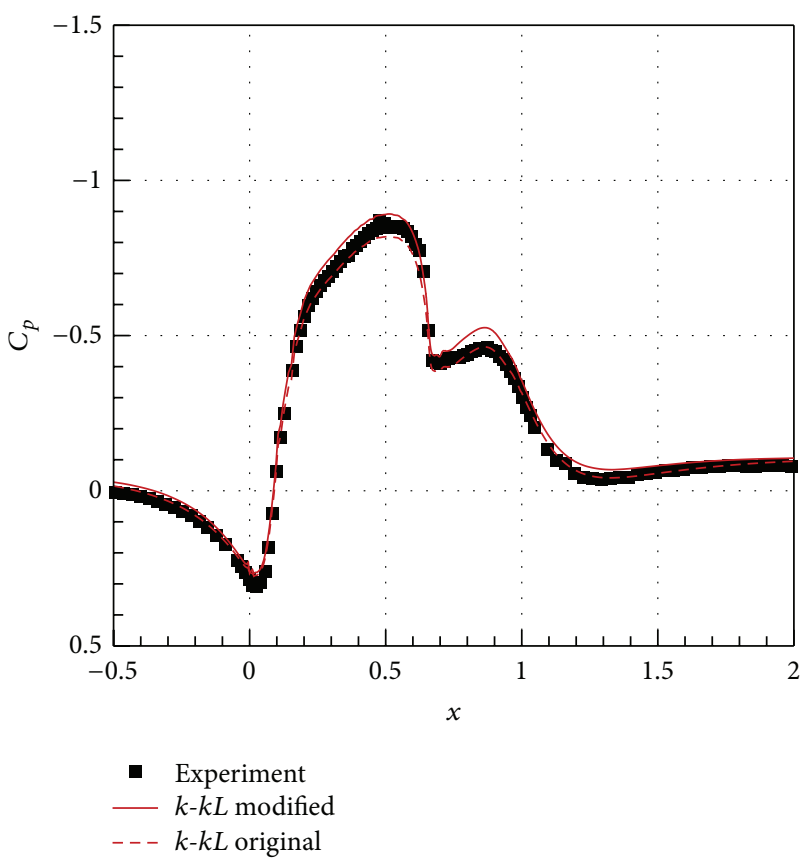

FIGURE 17: Surface pressure comparisons between experimental data and original and modified $k-k L$ simulations for the hump model case.

data compared to the original model, as seen in Figure 18. There are very large differences between the original model prediction and experimental data in the separation region. The original model predicted the correct size of the separation bubble compared to the modified model.

Figure 19 shows that there is a very small effect of grid resolution on the surface pressure distribution. The turbulence model comparisons in Figure 20 show that the $k$ - $k L$ turbulence model is slightly better than SST and $k-\varepsilon$ turbulence models as compared with experimental data.

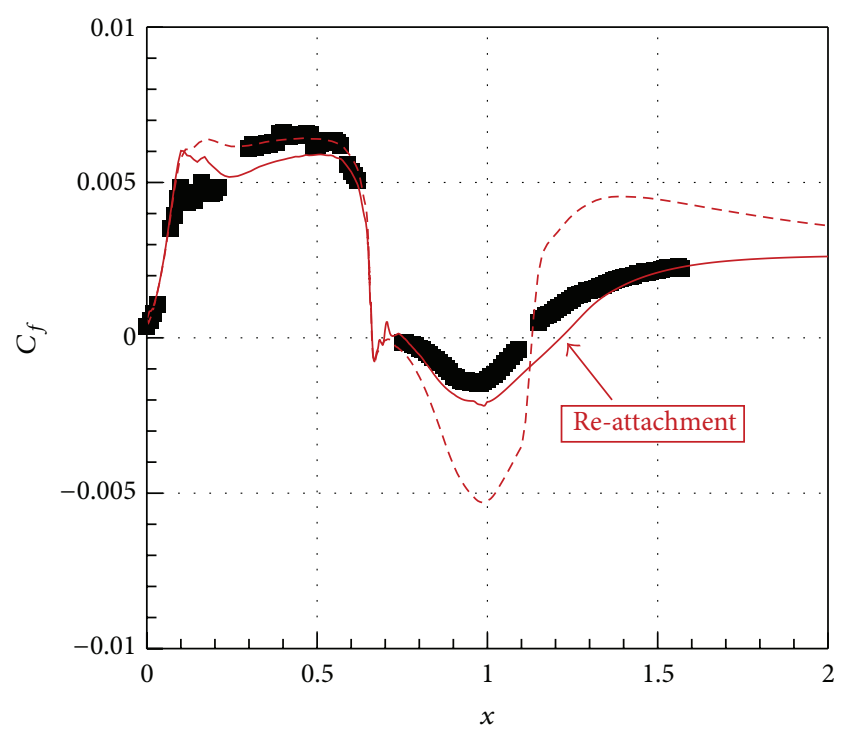

- Experiment

$-k-k L$ modified

$---k-k L$ original

FIGURE 18: Skin friction comparisons between experimental data and original and modified $k-k L$ simulations for the hump model case.

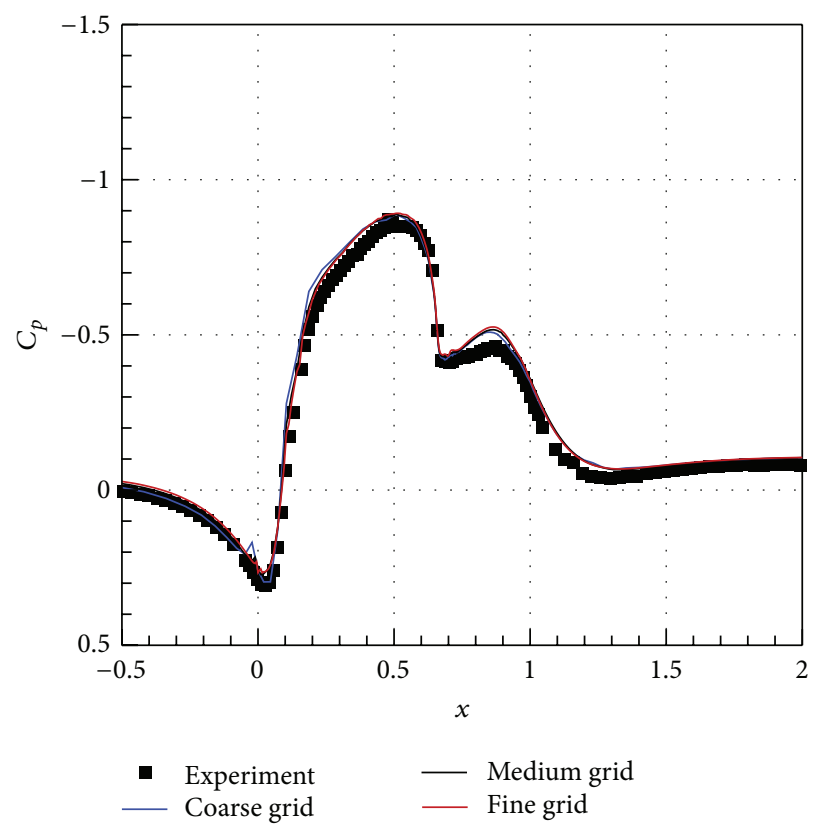

FIGURE 19: Surface pressure comparisons between experimental data and coarse, medium, and fine grid simulations for the hump model case.

Figure 21 shows that there is no effect of grid resolution on the skin friction results. The turbulence model comparisons in Figure 22 show that the $k-k L$ turbulence model is better than the $k-\varepsilon$ and SST turbulence models from the pressure recovery region aft as compared with experimental data. 


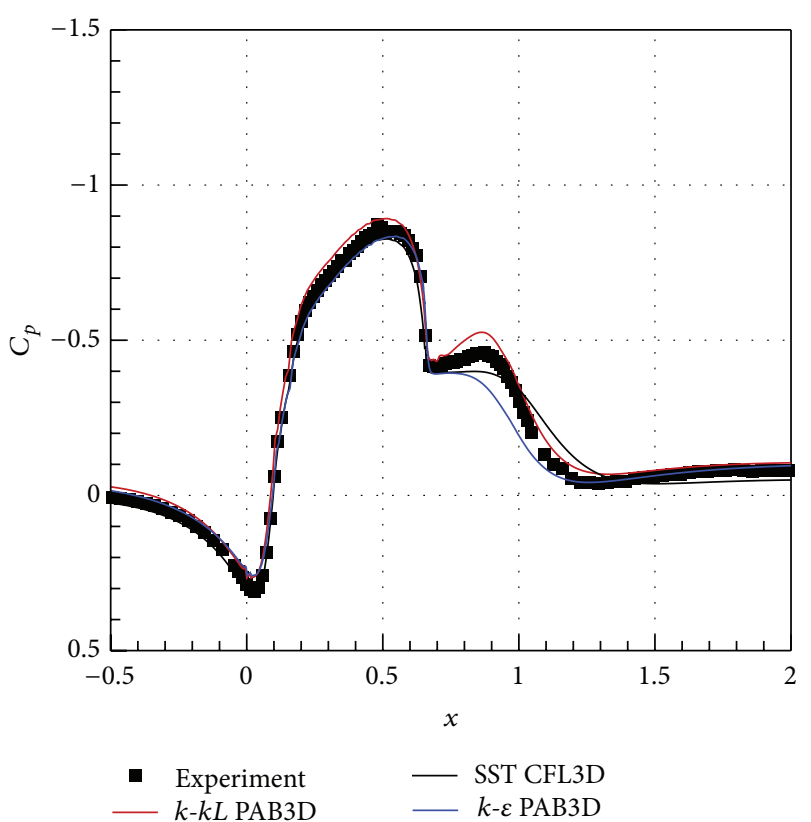

FIGURE 20: Surface pressure comparisons between experimental data and $k-k L$, SST, and $k-\varepsilon$ simulations for the hump model case.

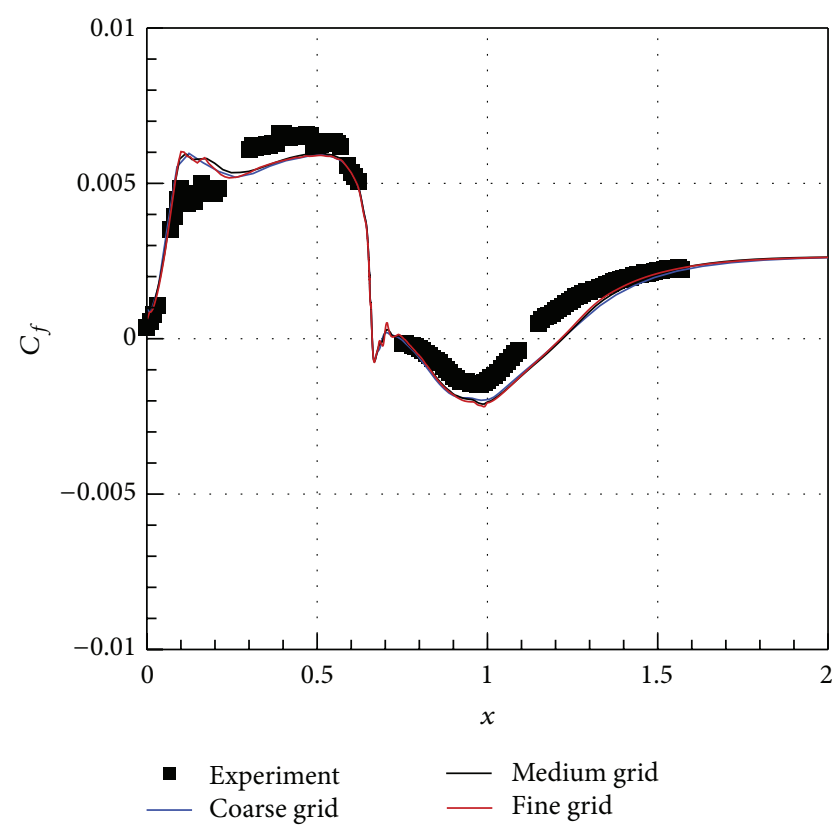

FIGURE 21: Skin friction comparisons between experimental data and coarse, medium, and fine grid simulations using $k-k L$ model for the hump model case.

3.4. Axisymmetric Subsonic Jet Case. The axisymmetric subsonic jet case validates the quality of the turbulence models' prediction of experimental data for shear flows. The experiment yielded measured velocities as well as turbulence quantities downstream of the jet exit using particle image velocimetry (PIV) [11]. Velocity and turbulence profiles of interest are measured at the centerline $(y=0)$. We are

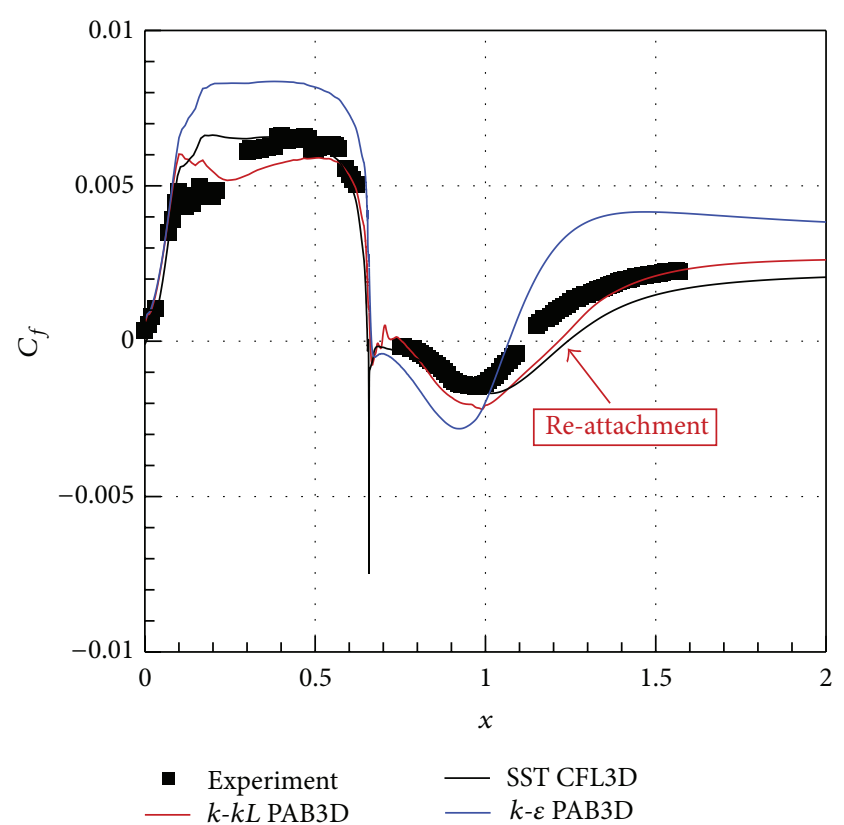

FIGURE 22: Skin friction comparisons between experimental data and $k-k L$, SST, and $k-\varepsilon$ simulations for the hump model case.

comparing the turbulence model results with the centerline values for velocity and turbulence data. The grid used is taken from [6], and a snapshot is shown in Figure 23. The grid is made of three blocks of $96 \times 96,60 \times 96$, and $256 \times 224$ cells. Block 1 is the interior of the nozzle, block 2 is the external flow-up to the nozzle, and block 3 extends from the exit of the nozzle to $x / D j=40 . D j$ is the diameter of the nozzle exit. Coarse, medium, and fine grid levels are used in the simulation where the coarser grid is exactly everyother-point of the next finer grid. It is important to note that this axisymmetric case is not a $2 \mathrm{D}$ computation; it uses a $5^{\circ}$ sector with one cell and symmetric boundary conditions on each side. $M_{\text {jet }}=u_{\text {jet }} / a_{\text {jet }}=0.51$, whereas the "acoustic Mach number," $u_{\text {jet }} / a_{\text {ref }}$, is approximately 0.5 . In the experiment, the axisymmetric jet exits into quiescent (nonmoving) air. However, because flow into quiescent air is difficult to achieve for some CFD codes, here the solution is computed with very low background ambient conditions $\left(M_{\text {ref }}=0.01\right.$, moving leftto-right, in the same direction as the jet).

First, we compared the three grid levels with the experimental data to validate the grids. Figure 24 shows the comparisons between the different grid levels using the $k-k L$ turbulence model and experimental data for the normalized centerline axial velocity. The nozzle centerline exit velocity is used to normalize the data. There is a very small difference between all grid level results. The prediction of the model is in fair agreement with experimental data; however, the jet core was predicted at around 7 diameters compared to experimental data of around 6. Also, the centerline velocity results show a slightly faster decay rate than the experimental data.

Figures 25 and 26 show the $k-k L$ model's ability to predict shear flow with other turbulence models. The jet core 


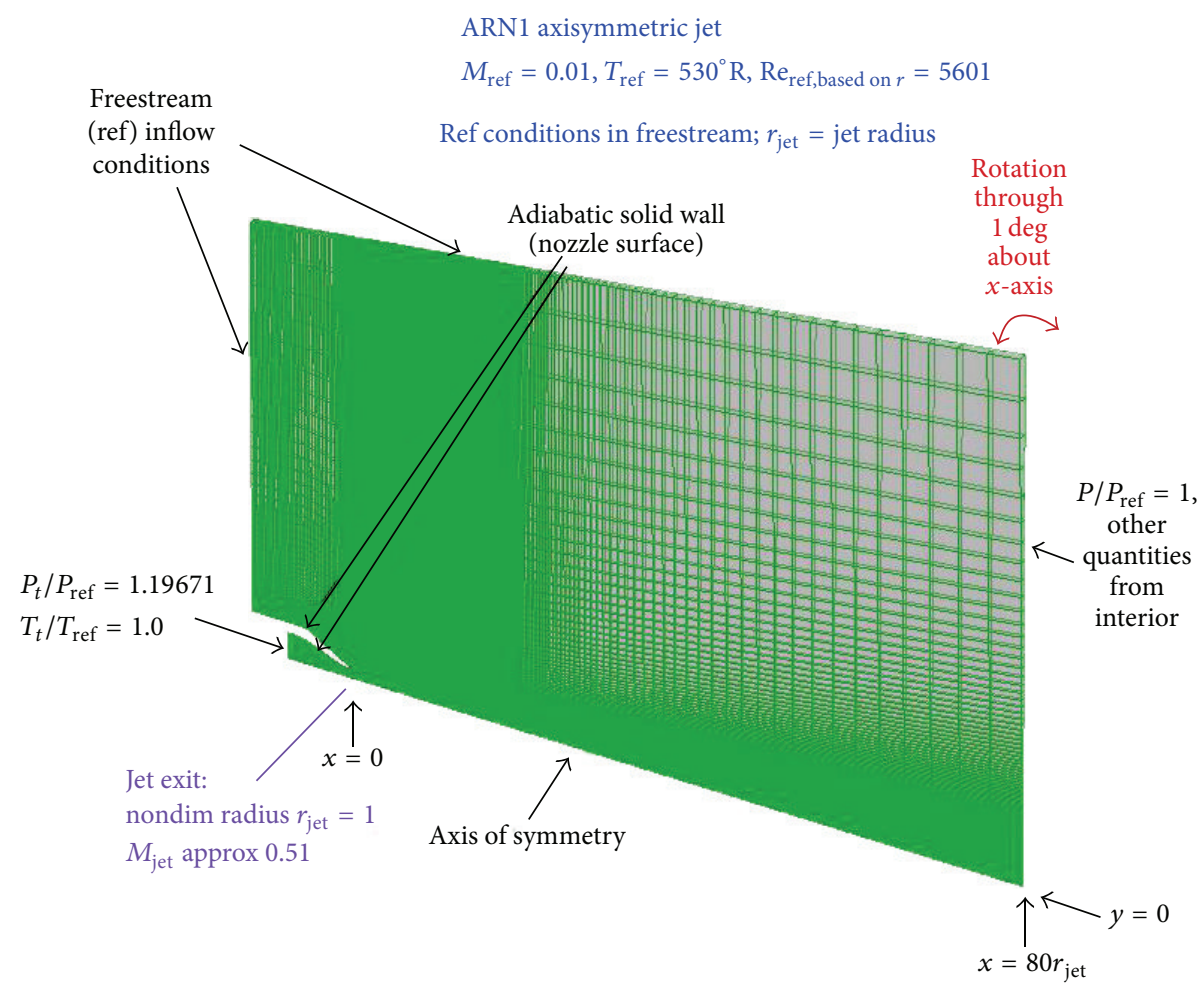

FIGURE 23: Grid distribution and flow conditions for subsonic jet flow case [6].

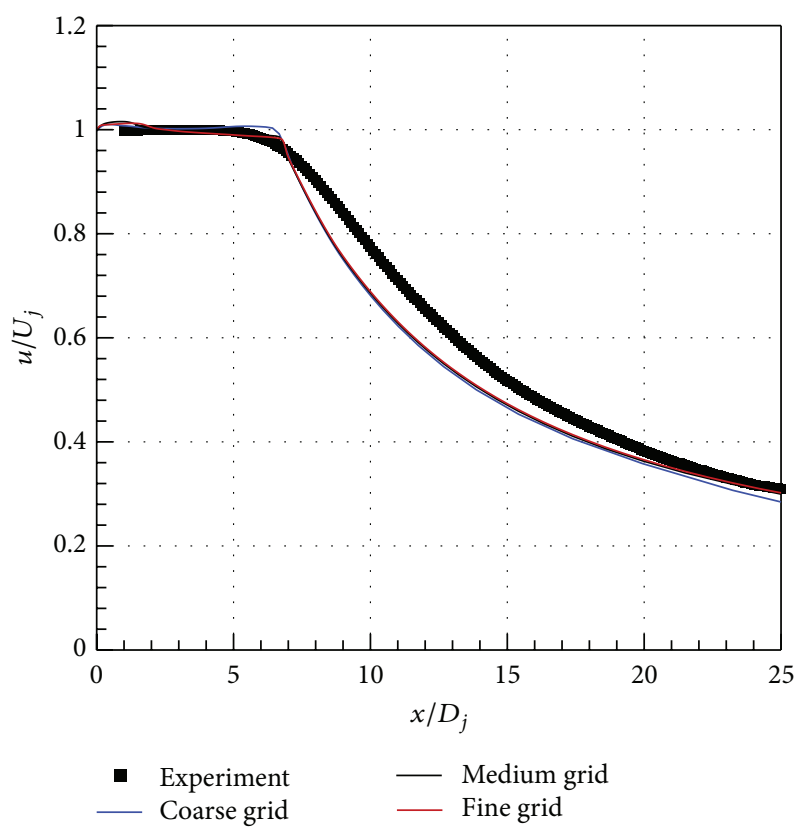

FIGURE 24: Grid effect on varations of centerline axial velocity using $k-k L$ turbulence model for the jet flow case.

length is better predicted with the $k-k L$ model compared to the $k-\varepsilon$ model, as shown in Figure 25. In Figure 26, both the SST and $k-\varepsilon$ models predict a long core length of around 8 as compared to a value of 7 predicted by

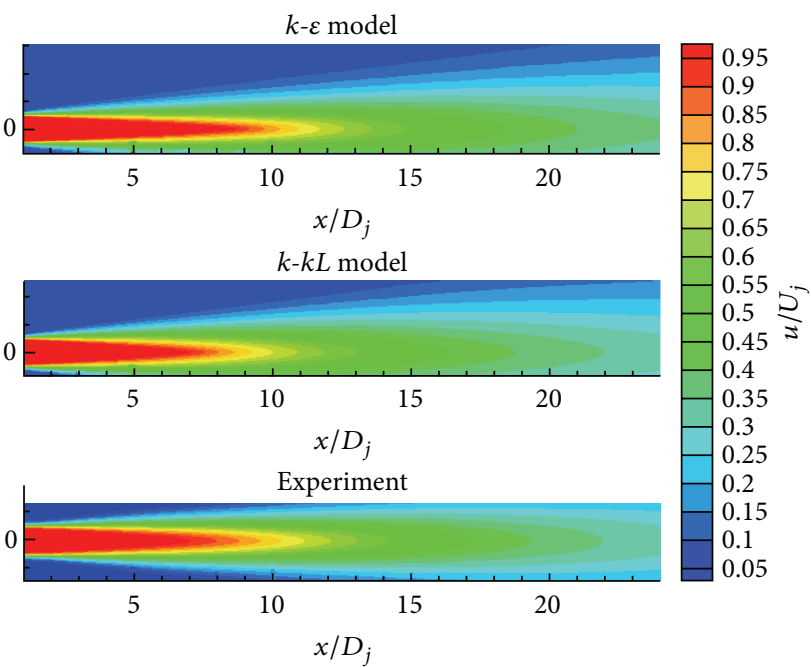

FIGURE 25: Normalized axial velocity comparisons between experimenal data and $k-k L$ and $k-\varepsilon$ turbulence models for the jet flow case.

the $k-k L$ model and the experimental data of around 6. The rate of decay predicted with the SST model is clearly the fastest as compared to the other models, and $k-k L$ predicted most closely with the experimental data in comparisons. The turbulence kinetic energy comparisons between turbulence models and experimental data are shown in Figure 27. The maximum value of kinetic energy is better predicted with the $k-k L$ model compared to the other models. The SST model 


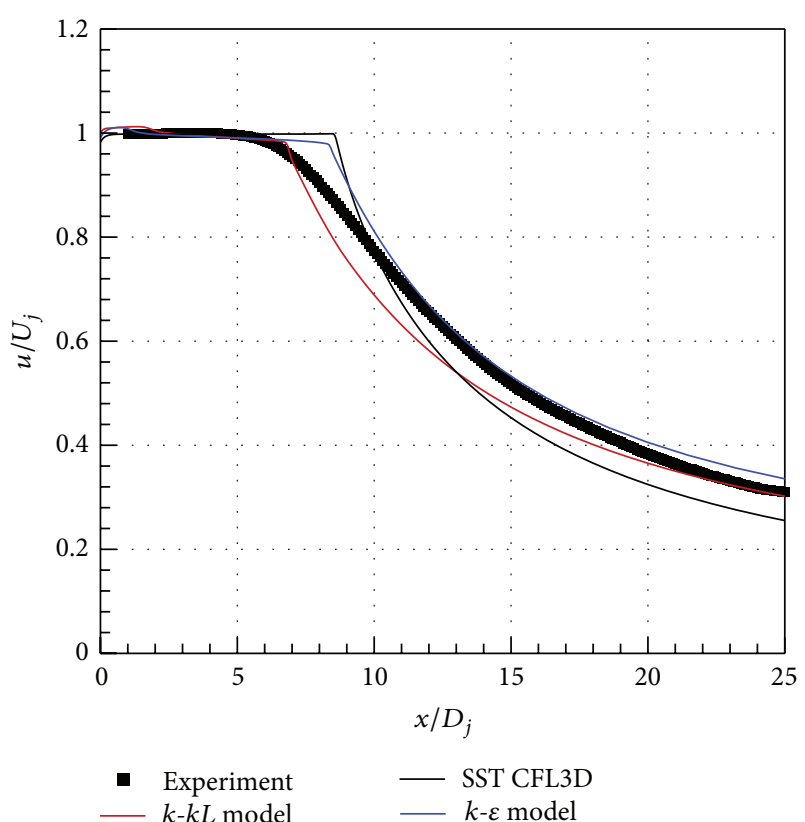

FIGURE 26: Axial centerline velocity comparisons between experimenal data and $k-k L$ and $k-\varepsilon$ turbulence models for the jet flow case.

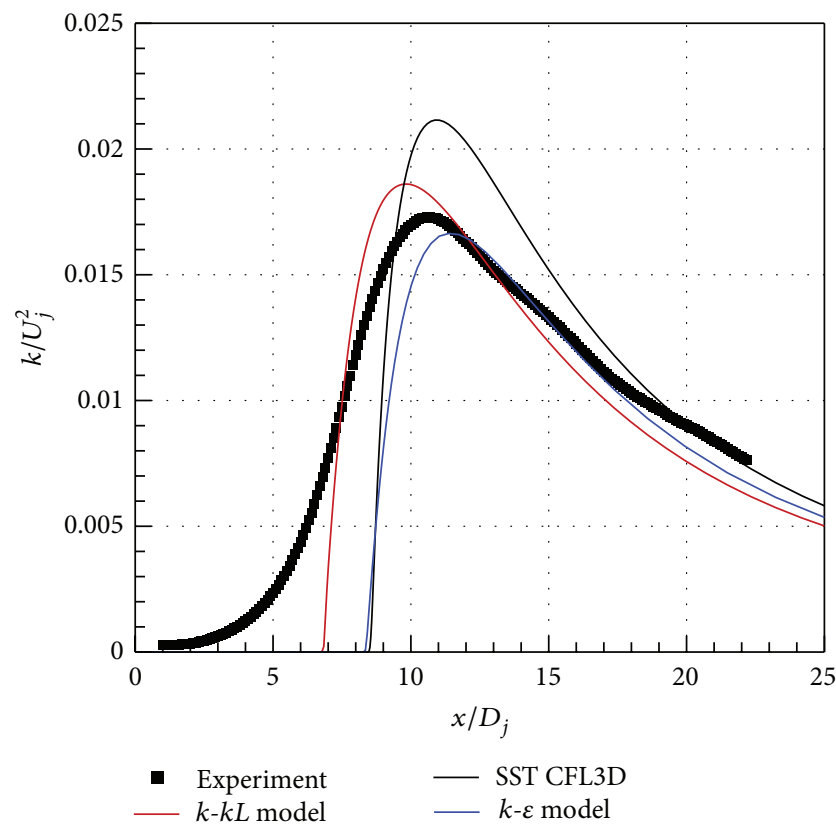

FIGURE 27: Turbulence kinetic energy comparisons between experimenal data and $k-k L$ and $k-\varepsilon$ turbulence models for the jet flow case.

overpredicted the turbulence kinetic energy level compared to the other models. All models failed to replicate the slow increase of turbulence kinetic energy for $x / D j<6$.

3.5. 2D Curved Backward-Facing Step Case. In this paper, we also compare turbulence models' predictions with LES CFD data provided by [6]. This LES case is for a $2 \mathrm{D}$ separating flow over a curved backward-facing step. The LES was run using

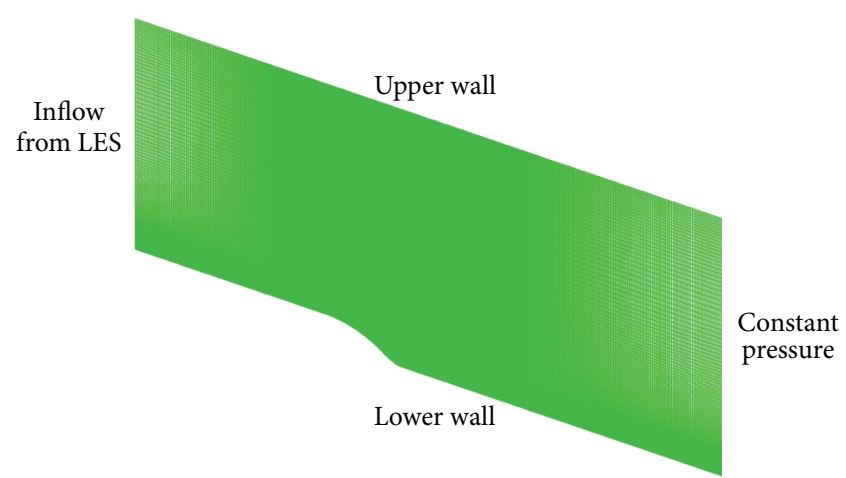

Figure 28: Grid distribution and flow conditions for 2D curved backward-facing step.

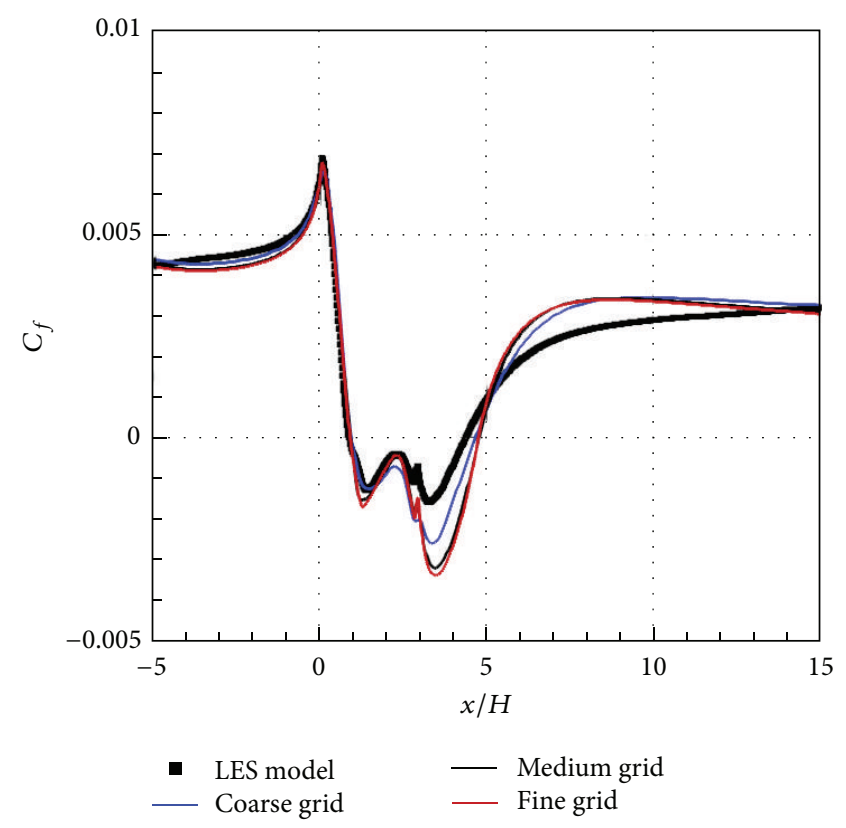

FIGURE 29: Skin friction comparisons between experimental data and coarse, medium, and fine grid simulations using $k-k L$ model for the $2 \mathrm{D}$ curved backward-facing step case.

an incompressible code and has been documented in several publications listed in [12]. Some relevant information is given here, but the interested reader is referred to [6] for complete details. The upstream duct height is $8.52 \mathrm{H}$, where $H$ is step height. The Reynolds number, based on $U_{\text {in }}$ and $H$, is 13700 . $U_{\text {in }}$ is the center-channel inlet velocity. At $x / H=-7.34$ data is extracted from LES solution [6].

The grid used in the present study is from [6]. This is a two-dimensional grid of $768 \times 160$ cells generated from the LES 23.6 million grid cells. The grid and boundary conditions are shown in Figure 28. The purpose of this comparison is to validate the ability of the $k-k L$ model to reproduce the LES data by enforcing LES boundary conditions for the inflow. The inflow boundary condition was extracted from the LES data. Figure 29 shows skin friction comparisons 


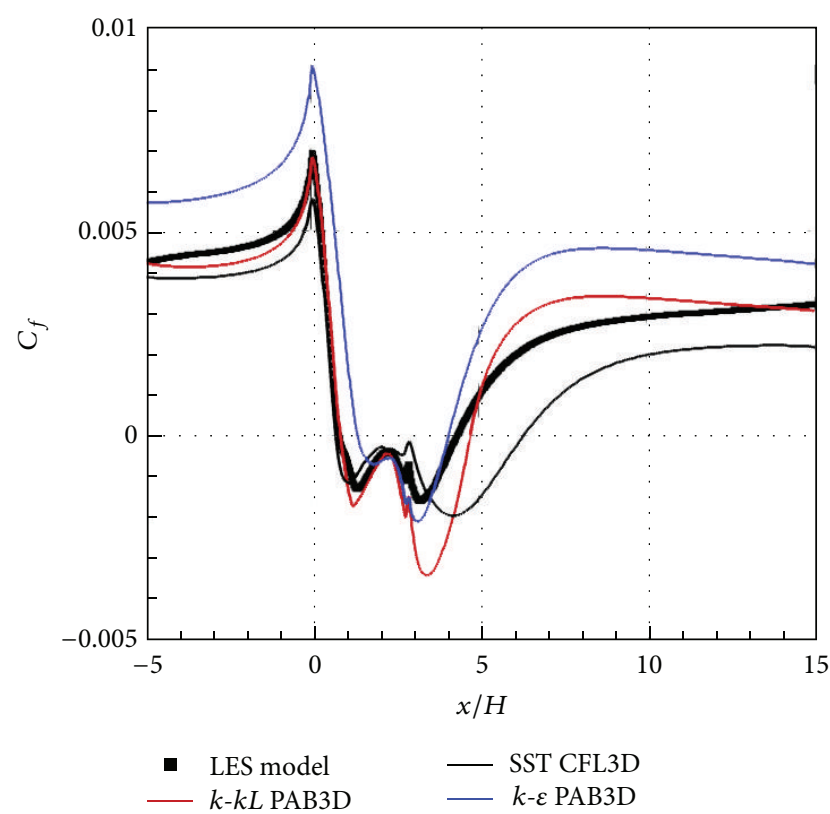

FIGURE 30: Skin friction comparisons between experimental data, and $k-k L$ and $k-\varepsilon$ simulations for the $2 \mathrm{D}$ curved backward-facing step case.

between the experimental data and the coarse, medium, and fine grid simulations using the $k-k L$ model. The skin friction variation is large between the coarse grid and the finest grid levels. The difference is very small between the medium and fine grid. Figure 30 shows the skin friction comparisons between experimental data and $k-k L$, SST, and $k-\varepsilon$ simulations. The level of skin friction predicted using $k-k L$ is in fair agreement with LES compared to either the SST or $k-\varepsilon$ predictions. Figure 31 shows the comparison between the LES and RANS turbulence model data separation and reattachment locations. In comparison with LES data, the $k-k L$ model predicted better separation and reattachment locations, as shown in Figures 30 and 31. The separation location, $X_{S} / H$, for LES is 0.82 , for $k-k L$ is 0.86 , for SST is 0.73 , and for $k-\varepsilon$ is 1.38 . The reattachment location, $X_{R} / H$, for LES is 4.35 , for $k-k L$ is 4.73 , for SST is 6.31 , and for $k-\varepsilon$ is 4.08 .

3.6. 2D Periodic Hill Case. The periodic hill data in this paper compared with the CFD data were provided by [6]. This LES case is for a $2 \mathrm{D}$ separating flow over periodic hills. The LES was run using an incompressible code and has been documented in several publications listed in [13]. The hill crest is $28 \mathrm{~mm}$, and the hills are separated by $L_{x}$ of $9 h$. The channel height, $L_{y}$, is $3.035 \mathrm{~h}$. The Reynolds number (based on $U_{b}$ and $h$ ) is 10595 . $U_{b}$ is the bulk velocity at the crest of the first hill. The flow is periodic in the streamwise direction.

The finest grid resolution used in this paper is $418 \times 194$ cells and is generated by [6]. Figure 32 shows the grid and boundary conditions. This comparison validates the ability of the $k-k L$ model to reproduce the LES data by enforcing the LES boundary condition for the inflow. The inflow boundary

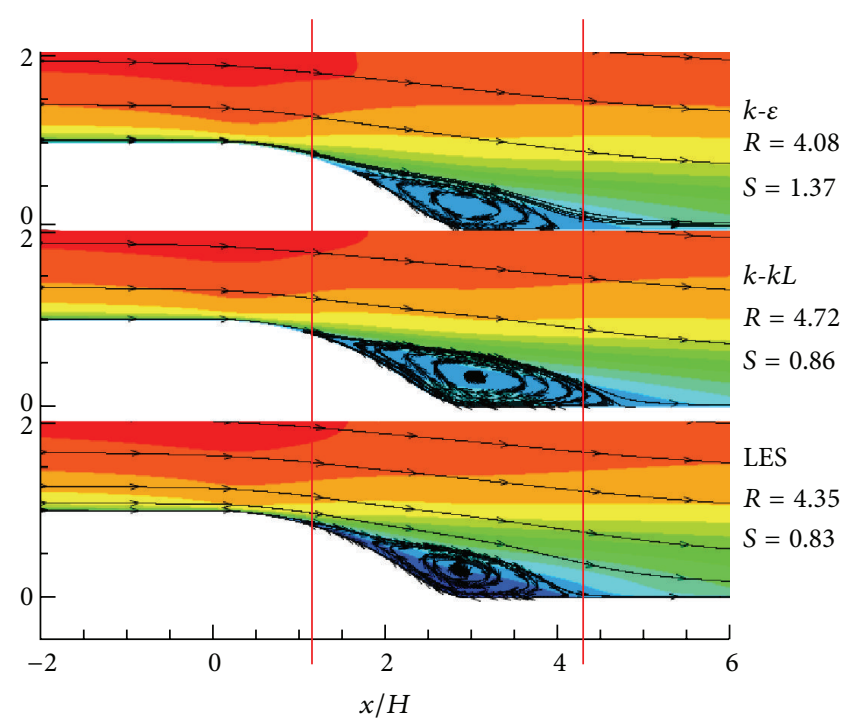

FIGURE 31: Comparison between LES and RANS turbulence models data separation and reattachment locations for the $2 \mathrm{D}$ curved backward-facing step case.

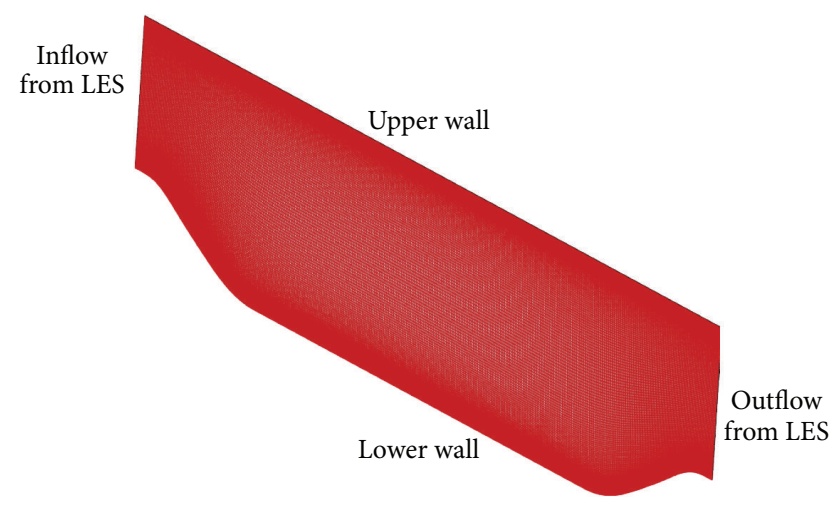

FIGURE 32: Grid distribution and boundary conditions for 2D periodic hill.

condition was extracted from the LES data. Figure 33 shows skin friction comparisons between experimental data and the coarse, medium, and fine grid simulations using the $k-k L$ model. The variation is quite large between the coarse grid and the finest grid levels. The difference is much smaller between the results using medium and fine grid. The separation bubble produced by the recent family of RANS models is in better agreement with LES data compared to the $k-\varepsilon$ results, as shown in Figures 34 and 35.

3.7. 2D NACA 4412 Airfoil. This section provides a validation case for turbulence models and data for comparison. For this particular "essentially incompressible" airfoil case with upper-surface trailing-edge separation [14], the data are from an experiment. The nominally 2D experiment utilized the NACA 4412 airfoil. For validation purposes, the definition of the airfoil shape is slightly altered so that the airfoil closes at chord $=1$ with a sharp trailing edge. Flowfield 


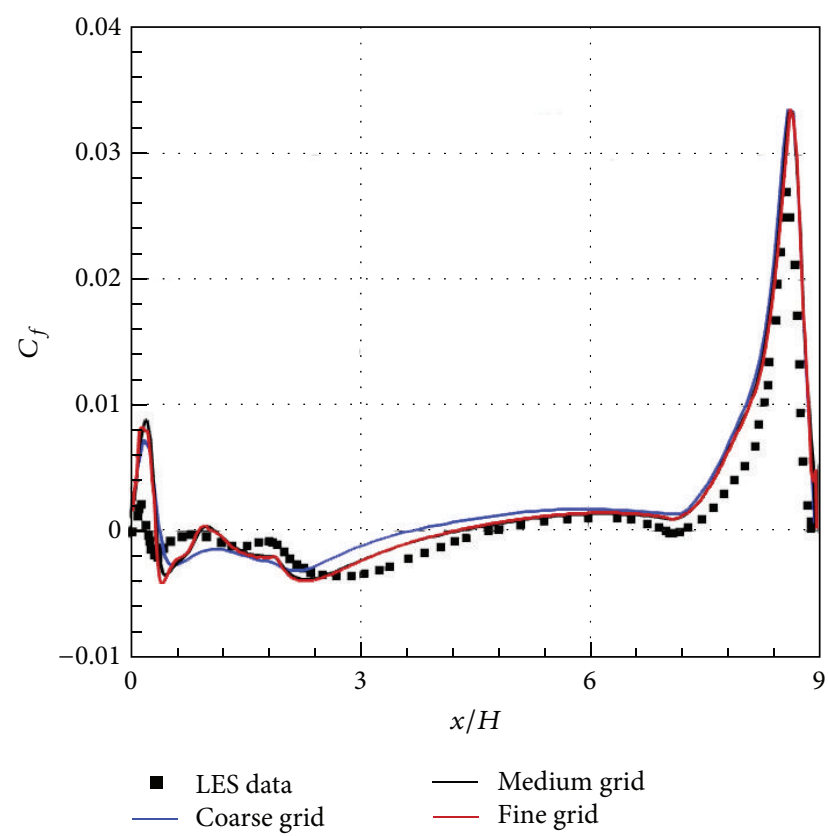

FIGURE 33: Skin friction comparisons between experimental data and coarse, medium, and fine grid simulations using $k-k L$ model for the $2 \mathrm{D}$ periodic hill case.

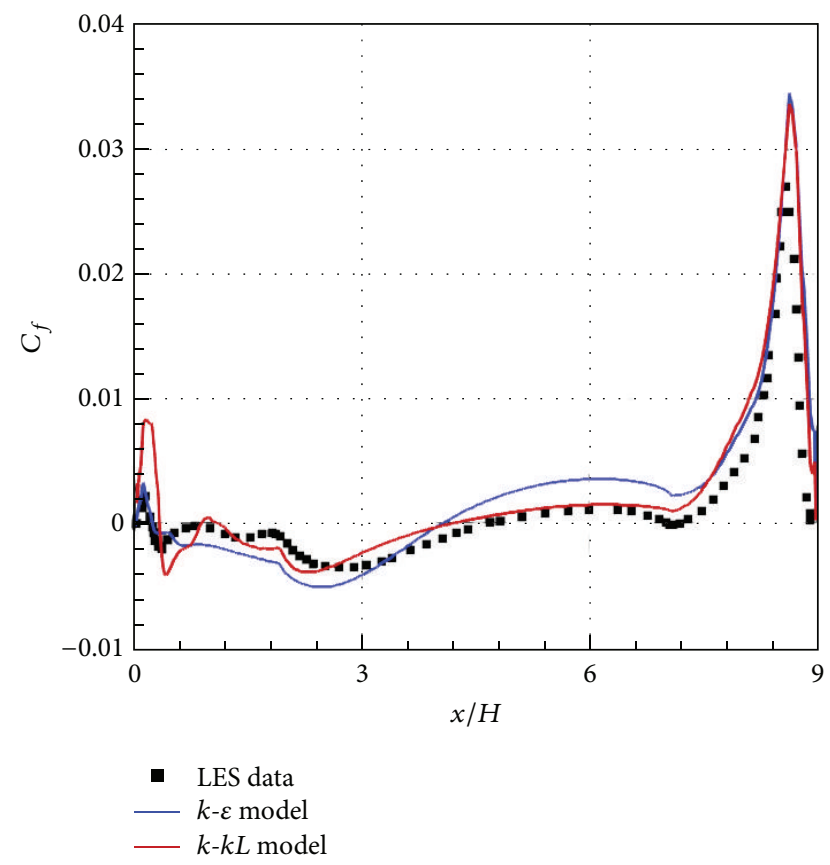

FIGURE 34: Skin friction comparisons between experimental data and $k-k L$, and $k-\varepsilon$ simulations for the $2 \mathrm{D}$ periodic hill case.

characteristics were measured with a flying hot-wire for the airfoil at 13.87 degrees angle of attack. The Reynolds number was 1.52 million per airfoil chord. Both the upper and lower boundary layers were tripped in the experiment $(2.5 \% c$ upper surface and $10.3 \% c$ lower surface). However, in the CFD, fully turbulent computations are performed. Also note
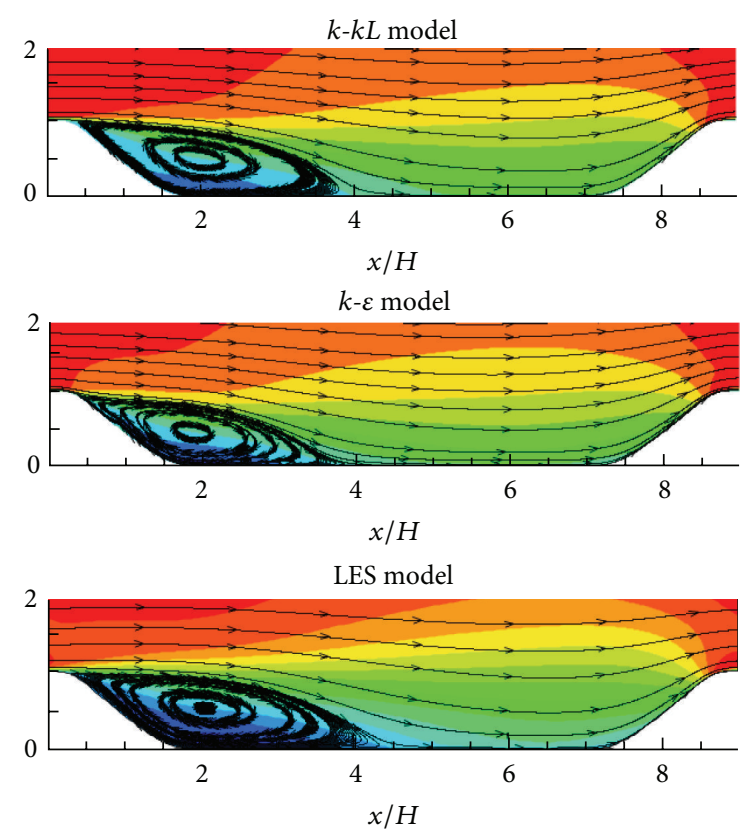

FIGURE 35: Comparison between LES and RANS turbulence model data separation and reattachment locations for the $2 \mathrm{D}$ periodic hill case.

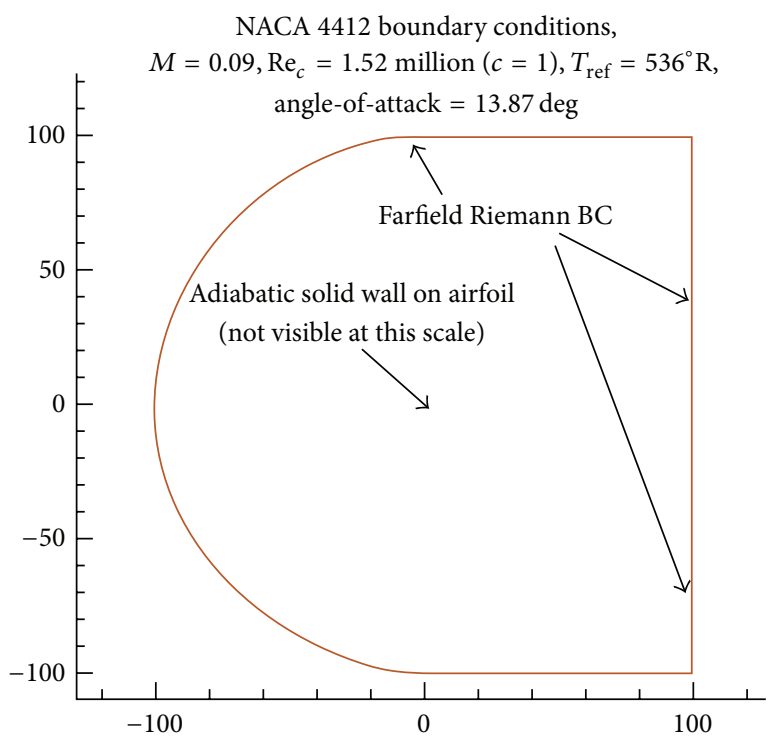

FIGURE 36: Flow conditions for NACA 4412 Airfoil [6].

that the CFD is performed here on grids with a farfield outer boundary extending to $100 c$, but the experiment was in a relatively small wind tunnel, which likely had some influence. Figure 36 shows the layout of the provided grids, along with typical boundary conditions. The experimental data for this case are provided at thousands of locations in the field surrounding the trailing edge region of the airfoil.

Figure 37 shows normalized $u$-velocity field data for NASA 4412 airfoil. The results from PAB3D are from the grid $(897 \times 257)$, which is at the fine grid level of the set provided in [6]. Figure 38 shows the calculated results 


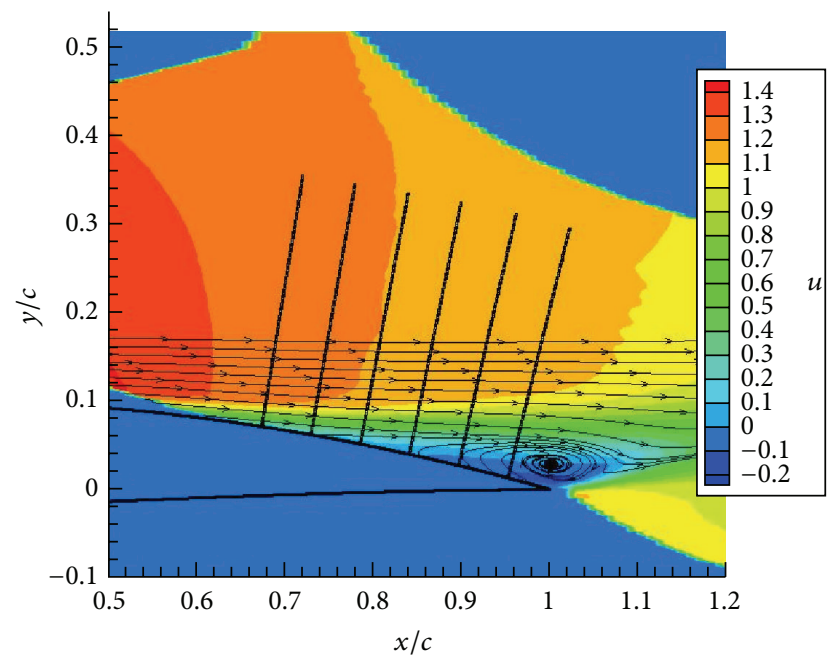

FIgURE 37: Experimental $u$-velocity for NASA 4412 Airfoil [6].

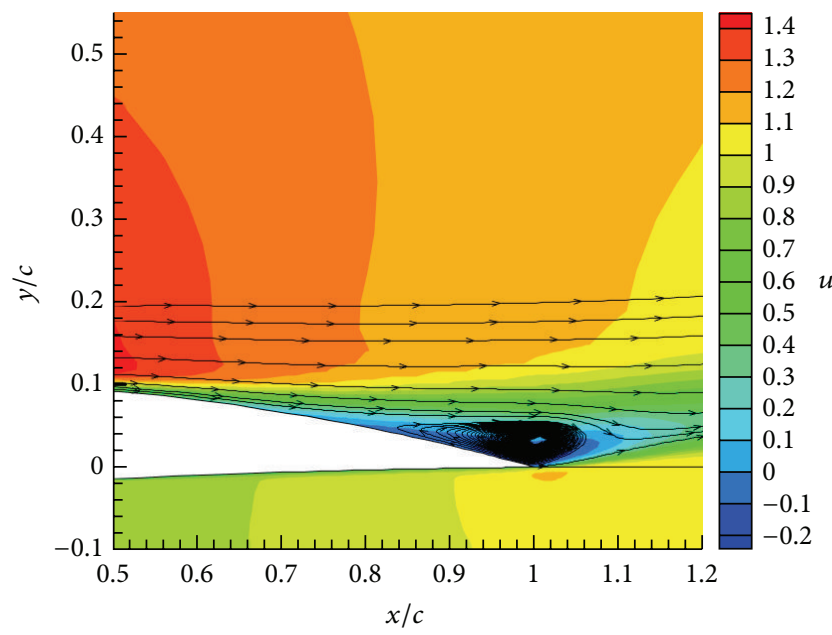

Figure 38: $k$ - $k L$ PAB3D $u$-velocity for NASA 4412 Airfoil.

using the $k-k L$ turbulence model which provides a separation flowfield similar to the measured data. In Figure 37, lines are also shown where (traditionally) researchers in the past have compared CFD results with this experiment: $x / c=$ $0.6753,0.7308,0.7863,0.8418,0.8973$, and 0.9528 . It is important to note that the experimental $u, v$, and $u^{\prime} v^{\prime}$ data were nondimensionalized with respect to a non-traditional velocity at a location only about 1 chord below and behind the airfoil. This is different from a traditional "freestream" value. As a result, $u / U_{\text {inf }}$ and $v / U_{\text {inf }}$ values from CFD need to be divided by roughly 0.93 in order to be comparable to the experimental normalization $u / U_{\text {ref }}$ (where $U_{\text {inf }}$ is the usual farfield freestream value and $U_{\text {ref }}$ is the experimental reference location). Similarly, $u^{\prime} v^{\prime} /\left(U_{\text {inf }}^{2}\right)$ turbulence values from CFD need to be divided by approximately $0.93^{2}$. There is little effect on the definition of surface pressure coefficient. Figure 39 shows the comparisons between turbulence model $u / U_{\text {ref }}$ results and measurement velocity data at different $x / c$ locations. The CFD results are in very good agreement with

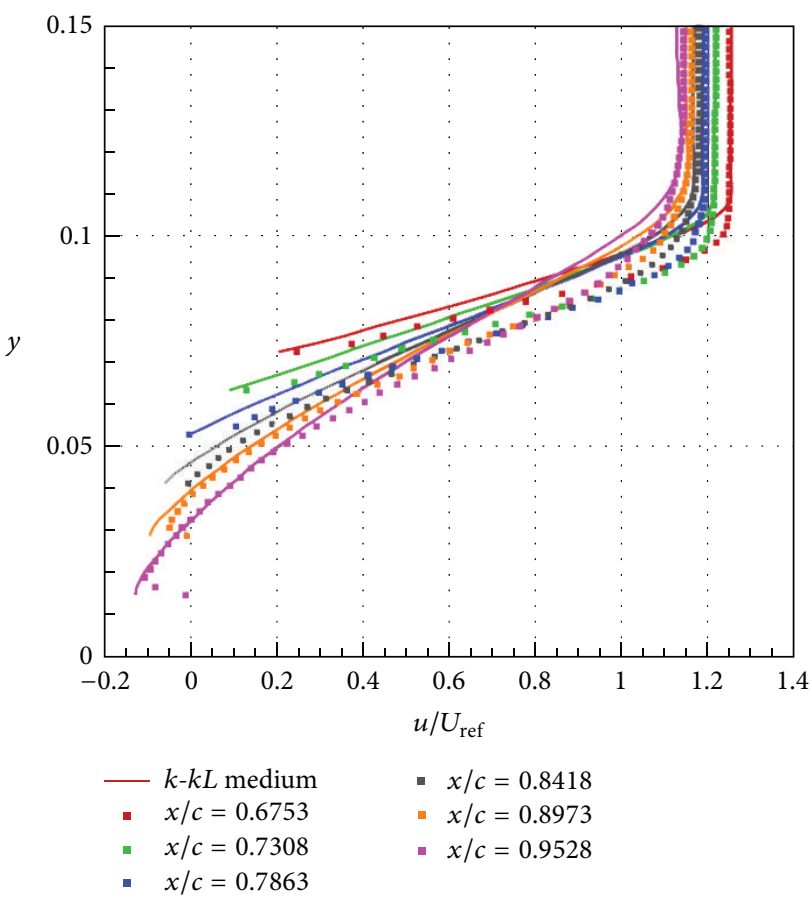

FIgURE 39: Experimental $u / U_{\text {ref }}$ velocity for NASA 4412 Airfoil.

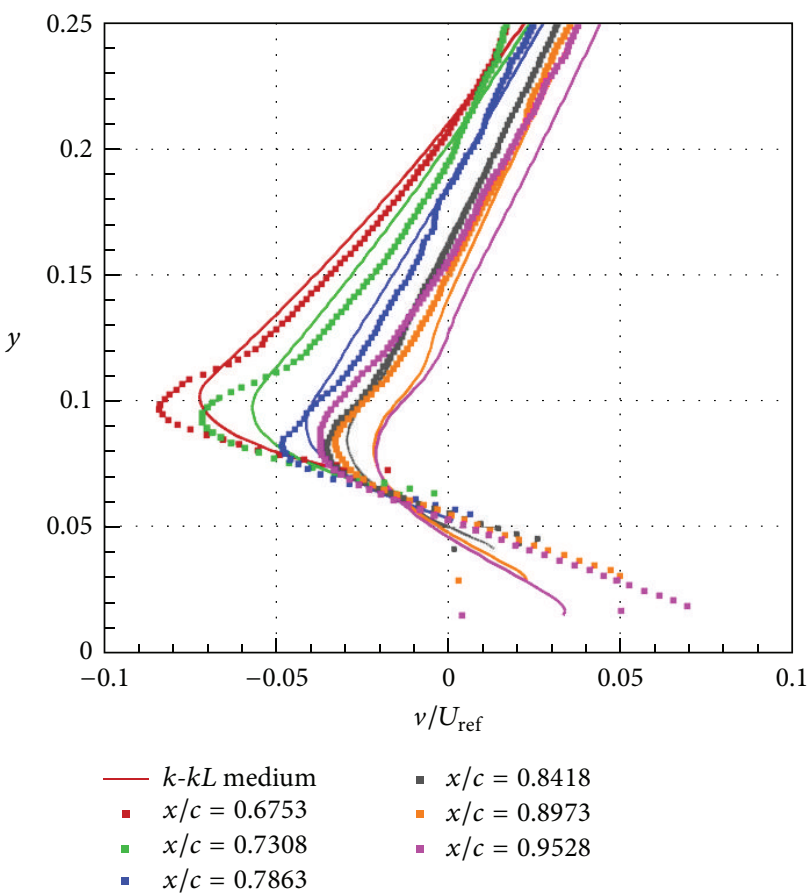

FIgURE 40: Experimental $v / U_{\text {ref }}$ velocity for NASA 4412 Airfoil.

the data. Similarly, the $v / U_{\text {ref }}$ results are in fair agreement with measured data as shown in Figure 40. However, as shown in Figure 41 the shear stress results underpredict the data for all $x / c$ locations. In general, the $k-k L$ results are similar or better than the calculations of other turbulence models documented in [6]. 


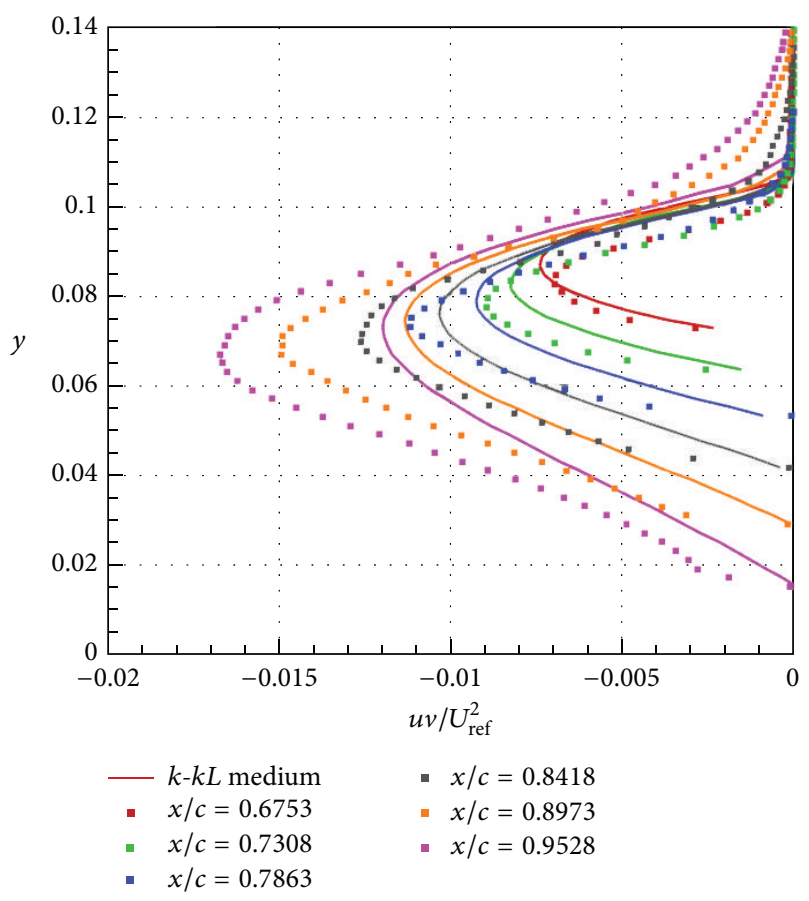

FIgURE 41: Experimental $u v / U_{\text {ref }}^{2}$ shear stress for NASA 4412 Airfoil.

3.8. Stationary Circular Cylinder in Cross Flow. The previous simulations have shown that the $k-k L$ turbulence models can produce steady state results for a wide range of flows and compared well with data and other turbulence models. One of the cases well-suited to demonstrate the unsteady capability of the model is the flow around a cylinder in cross flow. Figure 42 shows the grid topology used to simulate the 50,000 Reynolds number flow case with 24 blocks and 1.3 million grid points. A classical URANS model can produce threedimensional unsteady structures for this problem. However, the resolved structures are typically the size of the cylinder diameter as a result of the turbulent length scale in URANS, as shown in Figure 43, being proportional to the thickness of the shear layer and not proportional to the size of the resolved scales. The turbulent structures displayed behind the cylinder are similar to the ones observed using LES or hybrid RANS approaches similar to Partial Averaged Navier-Stokes (PANS) [17]. PANS is a hybrid RANS approach that acts as LES in the shear flow and wake regions. The $k-k L$ turbulence model has the same capability as the SAS approach used by Menter and demonstrated in several papers.

The unsteady solutions were averaged over the last 15,000 iterations (approximately 50 shedding cycles). The onset of asymmetric vortex shedding is seen to occur just after the first 60 time units. It was observed that approximately four sub-iterations per physical time step produced the optimal convergence per iteration. In the present results, four subiterations typically reduced the residual by three orders of magnitude at that time level, with no improvement using more iteration. The results were compared with the results using up to 20 sub-iteration, with no substantial difference in the final results. Figure 44 shows the centerline velocity comparison with experimental data [15]. The $k-k L$ turbulence

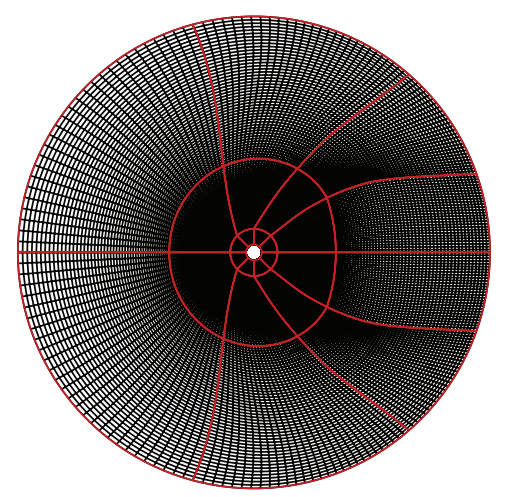

FIGURE 42: Grid topology for flow over cylinder.

model produces the best results when compared to $k-\varepsilon$, PANS, and measurement. Similar observations are noticed in the results shown in Figure 45 and Figure 46 for the data at $x / R=1$ and $x / R=3$, respectively.

\section{Concluding Remarks}

Most turbulence models use turbulence kinetic energy, which is a well-behaved equation. The second equation, representing the length scale, is considered the weakest link when using turbulence models including full Reynolds stress and hybrid RANS/LES formulations. The main objective of this paper is to find a better turbulence model with a more reliable second equation. Rotta shows that a reliable second equation can be formed in an exact transport equation for the turbulent length scale, $L$. Rotta's equation is well suited for a term-by-term modeling and shows some interesting features compared to other approaches. The most important difference is that the formulation leads to a natural inclusion of higher-order velocity derivatives into the source terms of the scale equation. The present paper assesses the results from a two-equation turbulence model, referred to as $k-k L$ and based on Menter's modification to Rotta's two-equation turbulence model.

Eight test cases were computed and presented covering an attached-flow flat plate case, five separate flow cases, a shear flow case, and an unsteady flow around a cylinder. The separated flow cases are the hump, the backward-facing step, the curved backward-facing step, periodic hill, and the flow around NACA 4412 airfoil. The flat plate case is compared with theoretical data. The hump, backwardfacing step, airfoil, and cylinder cases are compared with experimental data. The other cases are compared with LES data. The results are compared with other turbulence models such as SST and $k-\varepsilon$, and theoretical as well as experimental data. They demonstrate that the $k-k L$ model has the ability to produce results similar or better than SST results. In general, the $k-k L$ model gives much better results than the $k-\varepsilon$ turbulence model. The $k-k L$ model is best in predicting the size of the separation bubble (separation and re-attachment locations) than all the other turbulence models discussed in the present paper. The skin friction is better predicted 


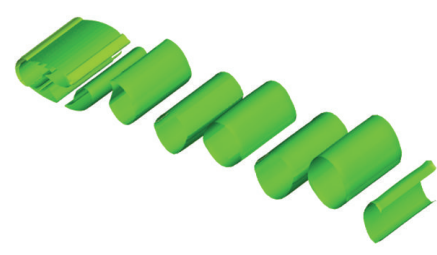

(a) $k-\varepsilon$

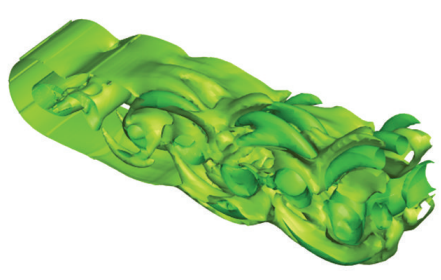

(b) $k-k L$

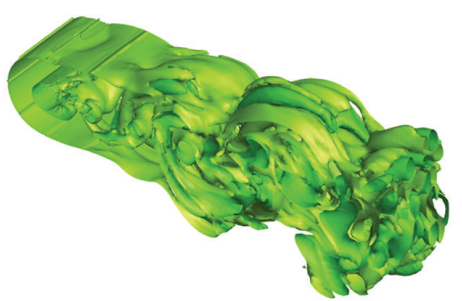

(c) PANS

FIGURE 43: Circular cylinder in a cross flow. Iso-surface colored by the $u$-velocity results from different turbulence models.

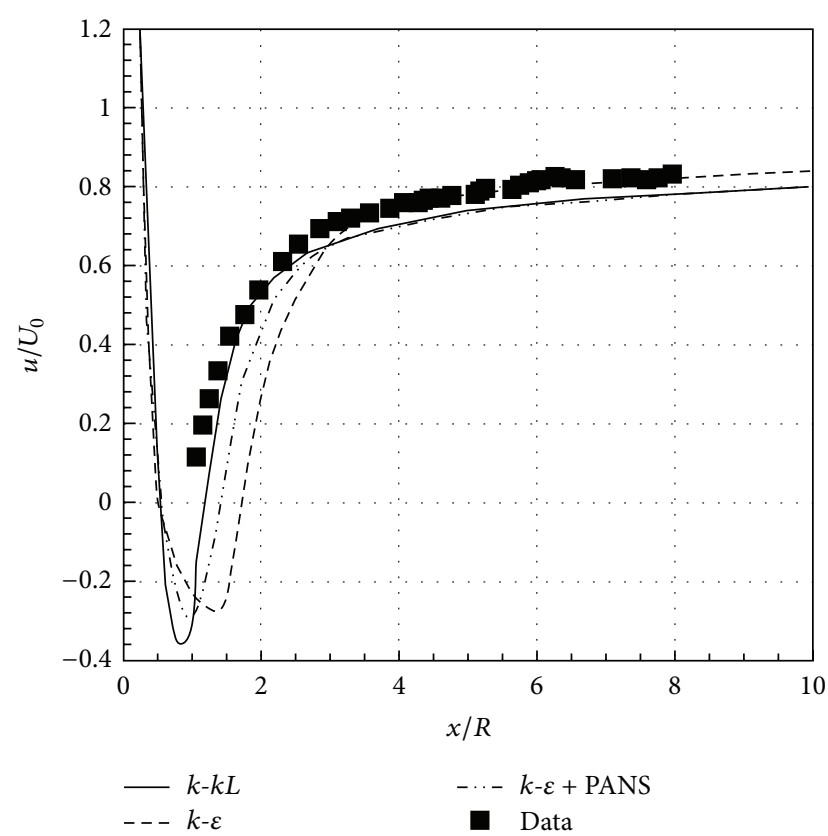

FIGURE 44: Time-averaged centerline $u$-velocity.

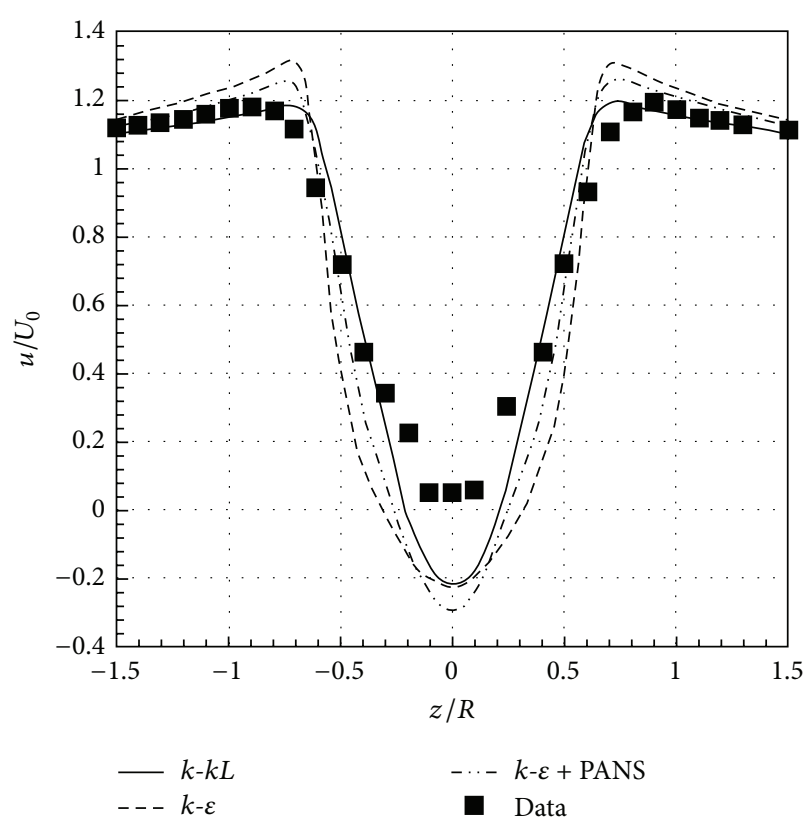

FIgURE 45: Time-averaged centerline $u$-velocity at $x / R=1$. by the $k-k L$ model for these cases than by other SST or $k-\varepsilon$ turbulence models. The $k-k L$ model also gives good agreement with the experimental data for the subsonic cold jet case as compared with the other turbulence models. The turbulent structures displayed behind the cylinder are similar to the ones observed using LES or hybrid RANS approaches similar to PANS.

All the results show grid convergence and independent solutions. Some of the results are compared with data from other two-equation turbulence models. In all the comparisons, the results from these models are computed at the fine grid level. In general, the results are as well as or better than the SST turbulence model and compared with available data. Future work will extend some of the present backward-facing cases to three-dimensional as well as other simple threedimensional cases.

\section{Nomenclature}

2D: Two-dimensional

$C_{f}$ : Skin friction coefficient
$C_{p}: \quad$ Pressure coefficient

$C_{\phi 1}$ : Production coefficient for the second equation

$C_{\phi 2}: \quad$ Dissipation coefficient for the second equation

$C_{\mu}: \quad$ Turbulence viscosity coefficient

$D j$ : $\quad$ Diameter of nozzle exit

$f_{c}$ : Compressibility function

$H$ : $\quad$ Step height

$h$ : Hill height

$k$ : $\quad$ Turbulence kinetic energy

$L: \quad$ Turbulence length scale

$L_{v k}: \quad$ von Karman length scale

$P_{k}: \quad$ Turbulence production

PANS: Partial Average Navier-Stokes

$U$ : Velocity

$U_{\text {ref }}:$ Reference velocity

$U_{\text {inf }}: \quad$ Farfield velocity

SAS: Scale-Adaptive Simulation

SST: $\quad$ Shear stress transport turbulence model

$u+: \quad$ Inner wall variable for streamwise velocity 


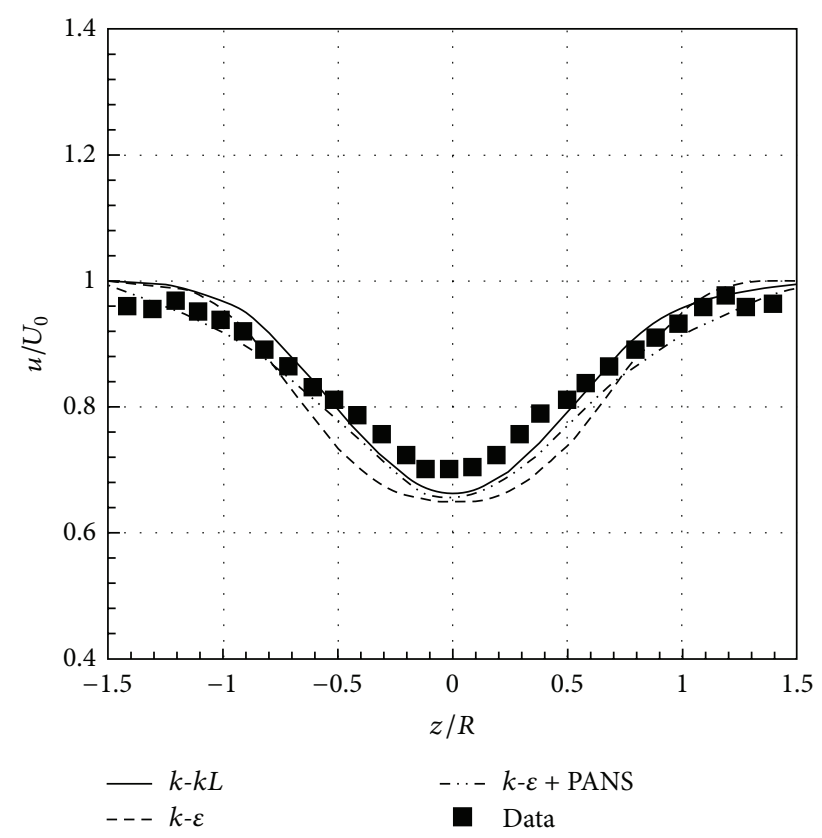

FIGURE 46: Time-averaged centerline $u$-velocity at $x / R=3$.

$y+: \quad$ Inner wall variable for distance

$\varepsilon$ : $\quad$ Turbulence dissipation

$\Phi: \quad$ Second turbulence equation variable

$\sigma_{k}$ : Diffusion coefficient for first equation

$\sigma_{\Phi}: \quad$ Diffusion coefficient for second equation

$\kappa: \quad$ von Karman constant

$\mu_{1}: \quad$ Laminar viscosity

$\mu_{t}: \quad$ T viscosity

$\rho: \quad$ Density

$\zeta_{1}, \zeta_{2}, \zeta_{3}$ : Constants for the second turbulence equation.

\section{Conflict of Interests}

The author declares that there is no conflict of interests regarding the publication of this paper.

\section{References}

[1] B. E. Launder and D. B. Spalding, Mathematical Models of Turbulence, Academic Press, London, UK, 1972.

[2] J. C. Rotta, "Statistische theorie nichthomogener turbulenz," Zeitschrift für Physik, vol. 129, pp. 547-572, 1951.

[3] F. R. Menter and Y. Egorov, "The scale-adaptive simulation method for unsteady turbulent flow predictions. part 1: theory and model description," Flow, Turbulence and Combustion, vol. 85, no. 1, pp. 113-138, 2010.

[4] Y. Egorov, F. R. Menter, R. Lechner, and D. Cokljat, "The scaleadaptive simulation method for unsteady turbulent flow predictions. part 2: application to complex flows," Flow, Turbulence and Combustion, vol. 85, no. 1, pp. 139-165, 2010.

[5] F. R. Menter, Y. Egorov, and D. Rusch, "Steady and unsteady flow modeling using the $k-\sqrt{k} L$ model," in Turbulence, Heat and Mass Transfer, vol. 5, 2006.
[6] Turbulence Model Validation Cases and Grids, http://turbmodels.larc.nasa.gov/.

[7] D. M. Driver and H. L. Seegmiller, "Features of a reattaching turbulent shear layer in divergent channel flow," AIAA Journal, vol. 23, no. 2, pp. 163-171, 1985.

[8] D. Greenblatt, K. B. Paschal, C.-S. Yao, J. Harris, N. W. Schaeffler, and A. E. Washburn, "Experimental investigation of separation control part 1: baseline and steady suction," AIAA Journal, vol. 44, no. 12, pp. 2820-2830, 2006.

[9] Hump Case and Grids, http://cfdval2004.larc.nasa.gov/ case3grids.html.

[10] D. Greenblatt, K. B. Paschal, C.-S. Yao, J. Harris, N. W. Schaeffler, and A. E. Washburn, "A separation control CFD validation test case, part 1: baseline and steady suction," AIAA Journal, vol. 44, no. 12, pp. 2820-2830, 2006.

[11] J. Bridges and C. Brown, "Parametric testing of chevrons on single flow hot jets," in Proceedings of the 10th AIAA/CEAS Aeroacoustics Conference, AIAA 2004-2824, 2004.

[12] Y. Bentaleb, S. Lardeau, and M. A. Leschziner, "Large-eddy simulation of turbulent boundary layer separation from a rounded step," Journal of Turbulence, vol. 13, no. 4, pp. 1-28, 2012.

[13] C. Melen, J. Frohlich, and W. Rodi, "Large Eddy simulation of the flow over periodic hills," in Proceedings of the 16th IMACS World Congress, Lausanne, Switzerland, 2000.

[14] D. Coles and A. J. Wadcock, "Flying-hot-wire study of flow past an NACA 4412 airfoil at maximum lift," AIAA Journal, vol. 17, no. 4, pp. 321-329, 1979.

[15] B. Cantwell and D. Coles, "An experimental study of entrainment and transport in the turbulent near wake of a circular cylinder," Journal of Fluid Mechanics, vol. 136, pp. 321-374, 1983.

[16] CFL3D description retreated from, http://cfl3d.larc.nasa.gov/.

[17] S. Girimaji and K. Abdol-Hamid, "Partially-averaged navier stokes model for turbulence: implementation and validation," in Proceedings of the 43rd AIAA Aerospace Sciences Meeting and Exhibit, Reno, Nev, USA, January 2005. 

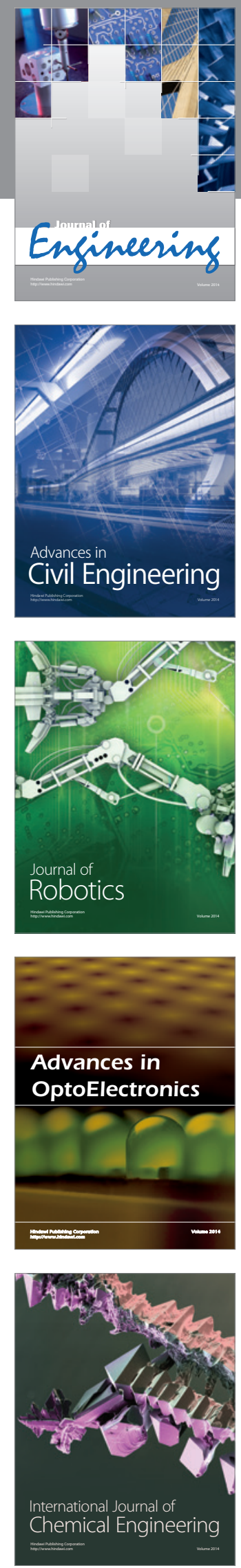

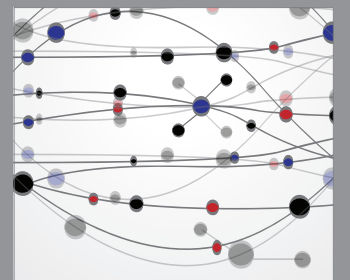

The Scientific World Journal
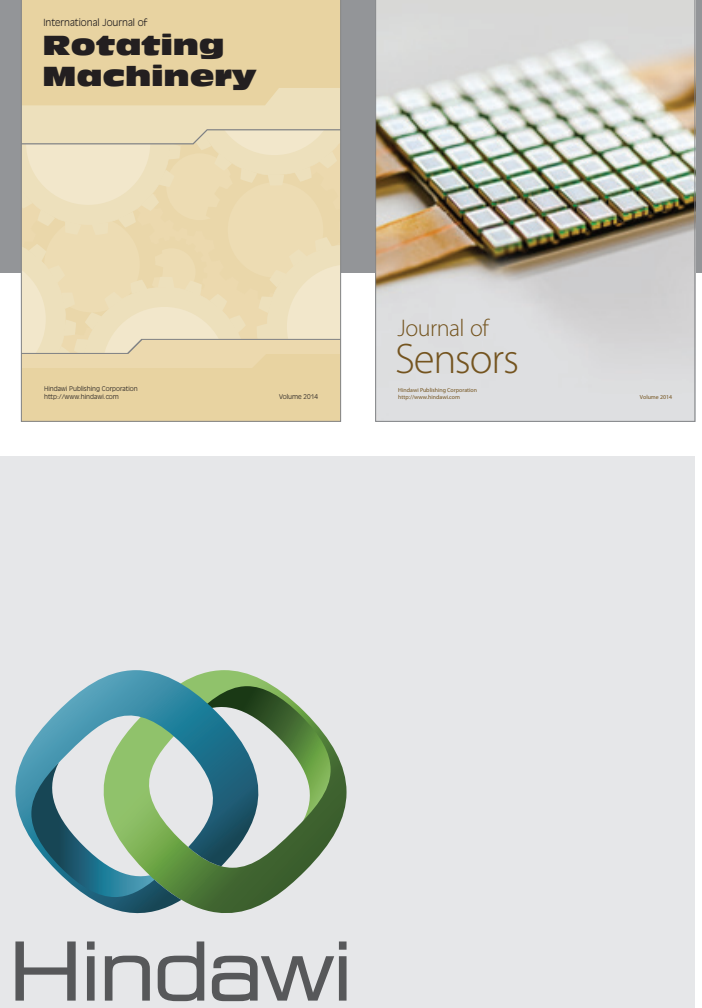

Submit your manuscripts at http://www.hindawi.com
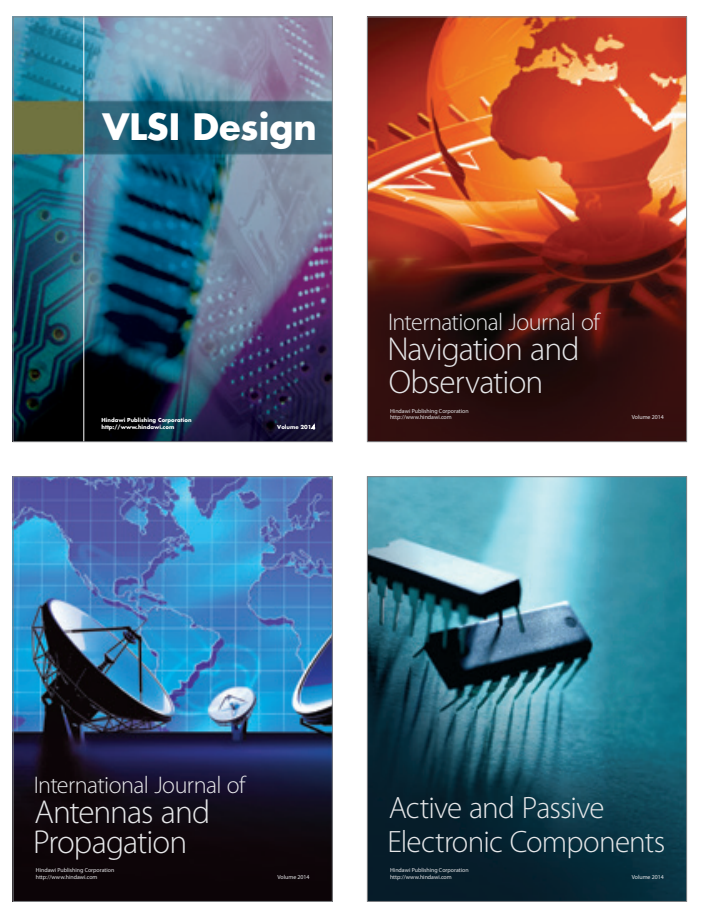
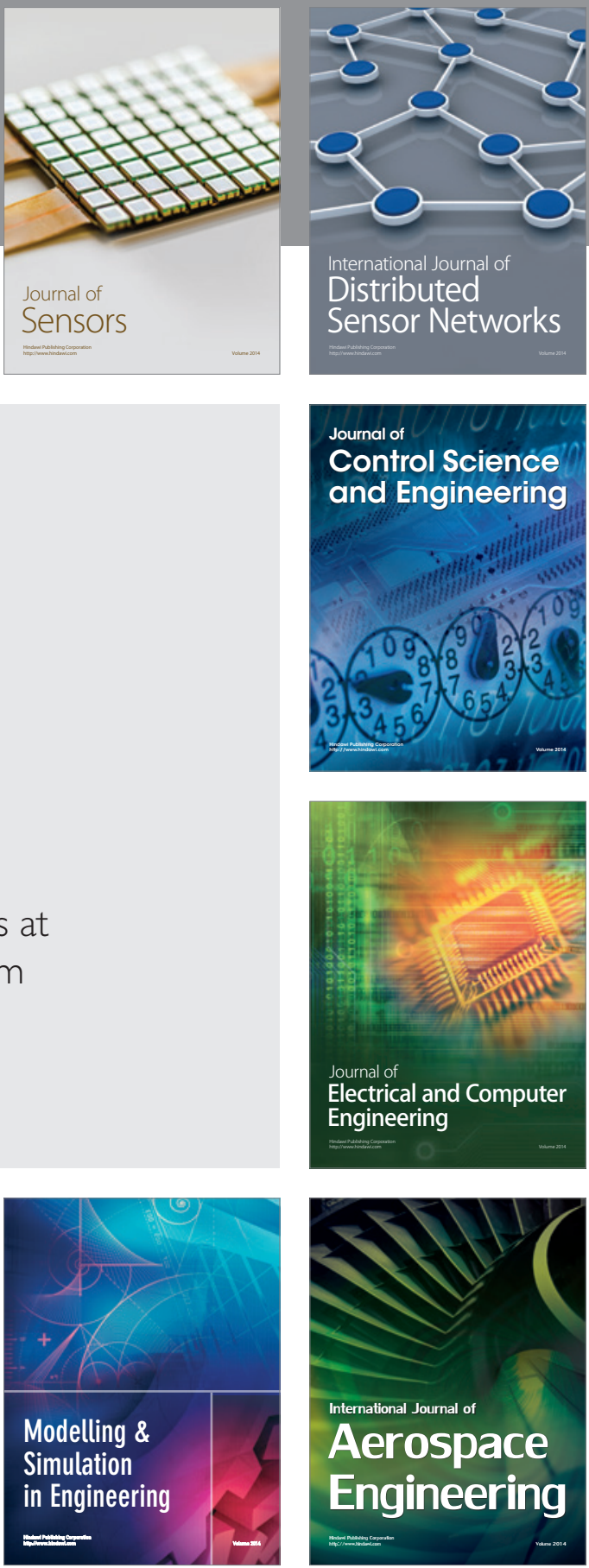

Journal of

Control Science

and Engineering
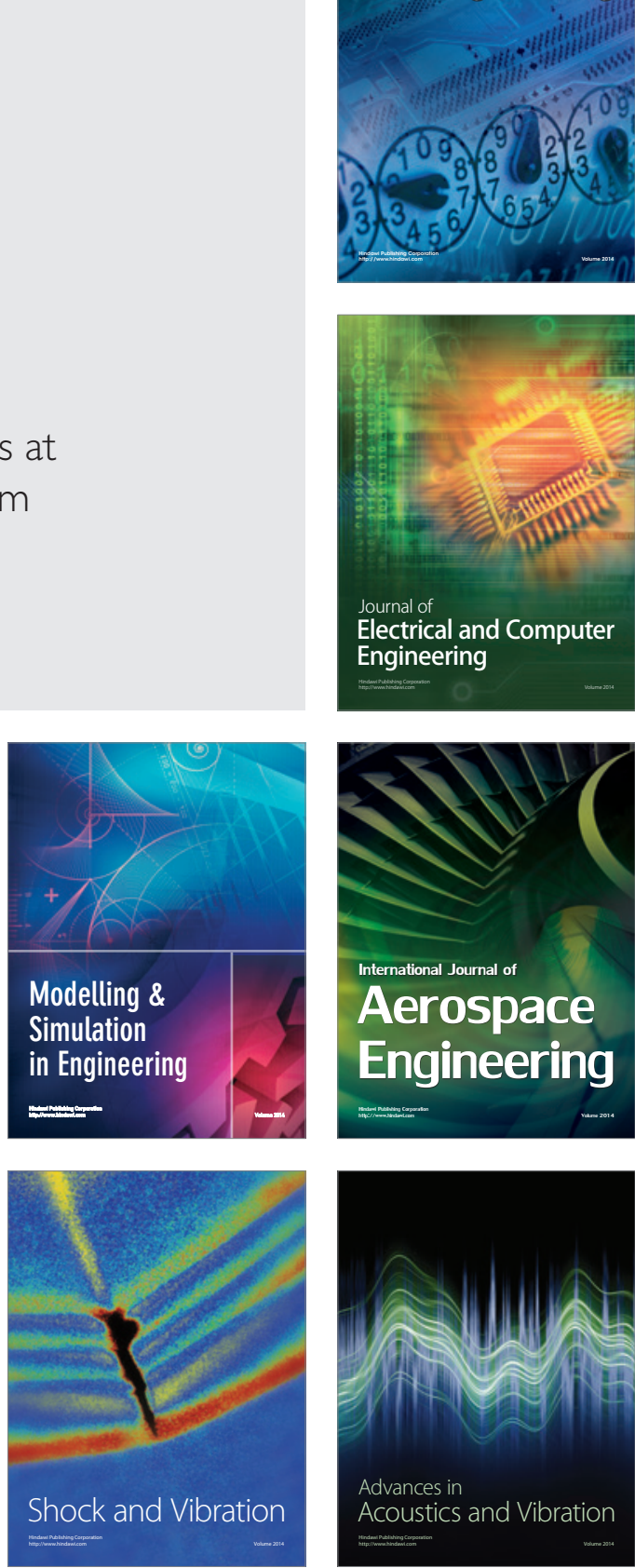